\title{
Harnack inequality for stable processes on $d$-sets
}

\author{
by \\ Krzysztof Bogdan, Andrzej Stós and Pawee Sztonyk (Wrocław)
}

\begin{abstract}
We investigate properties of functions which are harmonic with respect to $\alpha$-stable processes on $d$-sets such as the Sierpiński gasket or carpet. We prove the Harnack inequality for such functions. For every process we estimate its transition density and harmonic measure of the ball. We prove continuity of the density of the harmonic measure. We also give some results on the decay rate of harmonic functions on regular subsets of the $d$-set. In the case of the Sierpinski gasket we even obtain the Boundary Harnack Principle.
\end{abstract}

1. Introduction. In the last two decades we observed a rapid development of analysis and probability theory on fractals; see [DSV], [Str], [Ba], $[\mathrm{BB}],[\mathrm{BP}]$ and the references therein. [Ba], for example, presents probabilistic techniques in potential theory corresponding to the generator of the so-called fractional diffusion, the fractal analogue of the classical Brownian motion (see the next section for precise definitions).

Starting from the fractional diffusions, a class of subordinated processes on $d$-sets was introduced in [S]. By an analogy with the classical setting, we call these processes $\alpha$-stable. The definition is briefly recalled in Section 3 .

The present paper addresses several important problems of the potential theory of $\alpha$-stable processes on $d$-sets. One of the results is the Harnack inequality for nonnegative harmonic functions of the process. The main results were announced earlier in [BSS]. For related recent developments we refer the reader to $[\mathrm{FJ}],[\mathrm{FU}],[\mathrm{Kum}],[\mathrm{HL}]$.

The paper is organized as follows. In Section 3 we give estimates and some regularity results for the transition densities of the stable process. In Section 4 we obtain estimates for the expected exit time for subdomains of the $d$-set. We generally follow the approach designed in $[\mathrm{Ba}]$; in particular

2000 Mathematics Subject Classification: Primary 60J45; Secondary 60J35.

Key words and phrases: stable processes, fractals, diffusions on fractals, Harnack inequality, Boundary Harnack Principle.

Research partially supported by KBN grant 2 P03A 04122 and RTN Harmonic Analysis and Related Problems contract HPRN-CT-2001-00273-HARP. 
Lemmas 4.1 and 4.3, and Proposition 4.4 have their analogues in the diffusion case. Here we give new and slightly generalized computations related to the $\alpha$-stable process.

In Section 6 we prove the existence and joint continuity of the Poisson kernel $P_{D}(x, y)$ for an open ball $D$ in the $d$-set. $P_{D}(x, y)$ is given by the Ikeda-Watanabe Formula [IW] describing a relation between the harmonic measure and the Lévy measure which is absent in the diffusion case. We derive optimal estimates for the Poisson kernel when $x$ and $y$ are away from the boundary (Proposition 6.4). The estimates turn out to be sufficient for the proof of the Harnack inequality when $\alpha \in\left(0,2 / d_{w}\right) \cup\left(d_{s}, 2\right)$. The latter is given in Section 7. In the recurrent case the proof of Lemma 7.3 employs an interesting formula (56) involving the hitting time for a point and the Green function. The transient case relies on estimates of the Poisson kernel for balls. We note here that the Harnack inequality has been recently established for fractional diffusions ([Ba], [BB1]) and for pure jump processes in $\mathbb{R}^{N}([\mathrm{BL}])$. In each case, including ours, the methods of proof are completely different.

Section 8 gives an estimate for the exit times of subdomains of the $d$-set (Theorem 8.3) which easily yields the decay rate of harmonic functions near the boundary (Theorem 8.4). The latter has an analogue in the theory of rotation invariant $\alpha$-stable processes in $\mathbb{R}^{N}$ (see [B, Lemma 3]). However, [B] makes an essential use of the exact formula for the Poisson kernel for a ball, which is not available in our case. Section 8 also contains a Carleson type estimate for $\alpha \in\left(0,2 / d_{w}\right)$ with a proof adapted from [BBy]. Our main contribution is in showing that the weak scaling of the process is sufficient for this proof to work. The restriction on $\alpha$ above is due to the fact that our proof depends on the polarity of the boundary of a ball. Finally, we give a proof of the Boundary Harnack Principle in the Sierpiński gasket case for $\alpha \in\left(0,2 / d_{w}\right) \cup\left(d_{s}, 2\right)$. Due to the simple geometry of this set the proof is an application of the Harnack inequality. We believe that the Boundary Harnack Principle holds more generally (e.g. for the Sierpiński carpet) but more complicated methods must be used to prove it.

2. Preliminaries. In this section we collect some notation and definitions adapted from $[\mathrm{Ba}]$ and $[\mathrm{P}]$.

Let $F$ be a nonempty closed subset of $\mathbb{R}^{N}, N \geq 1$. Set $d \in(0, N]$. We say that a (positive) Borel measure $\mu$ is a $d$-measure on $F$ if for some constants $c_{1}, c_{2}>0$ it satisfies

$$
c_{1} r^{d} \leq \mu(B(x, r)) \leq c_{2} r^{d}, \quad x \in F, 0<r \leq r_{0},
$$

where $r_{0}$ is the diameter of $F$ and $B(x, r)$ denotes the ball in $\mathbb{R}^{N}$ with center $x$ and radius $r$. We call $F$ a $d$-set if $F=\operatorname{supp}(\mu)$ for some $d$-measure $\mu$. It is known that any $d$-measure is a regular Borel measure. Any two $d$-measures 
on the same $d$-set $F$ are equivalent and the $d$-dimensional Hausdorff measure restricted to a $d$-set $F$ is a $d$-measure [JW].

We use $c$ (with subscripts) to denote positive and finite constants which depend only on the $d$-measure $\mu, F$ (and $d$ ), the fractional diffusion on $F$ and the stability index $\alpha$ (see below). Any additional dependence is indicated explicitly, e.g. $c_{4}=c_{4}(D, \kappa)$. Constants are numbered consecutively within each proof. The value of $c$ (without subscript) may change from place to place. We write (e.g.) $f(x) \asymp g(x), x \in F$, to indicate that there are constants $c_{1}, c_{2}>0$ (independent of $x$ ) such that $c_{1} f(x) \leq g(x) \leq c_{2} f(x)$ for all $x \in F$. We denote by $|\cdot|$ the Euclidean distance in $\mathbb{R}^{N}$. From now on $B(x, r)$ denotes the Euclidean ball intersected with our $d$-set $F$. For a subset $D \subseteq F$ we always take complements in $F$, i.e. $D^{\mathrm{c}}=F \backslash D$. Without losing generality, in what follows we assume that $0 \in F$, which often simplifies the notation.

The following lemma is a convenient replacement for integration in polar coordinates.

Lemma 2.1. Let $F$ and $\mu$ be as introduced above. Then for every $x_{0} \in F$, $r>0$ and $\lambda>0$ there is $c=c(\lambda)$ such that

$$
\begin{gathered}
\int_{\left|x-x_{0}\right|>r}\left|x-x_{0}\right|^{-d-\lambda} d \mu(x) \leq c r^{-\lambda}, \\
\int_{\left|x-x_{0}\right|<r}\left|x-x_{0}\right|^{-d+\lambda} d \mu(x) \leq c r^{\lambda} .
\end{gathered}
$$

Proof. From (1) it follows that

$$
\begin{aligned}
\int_{\left|x-x_{0}\right|>r}\left|x-x_{0}\right|^{-d-\lambda} d \mu(x) & =\sum_{n=0}^{\infty} \int_{2^{n} r<\left|x-x_{0}\right| \leq 2^{n+1} r}\left|x-x_{0}\right|^{-d-\lambda} d \mu(x) \\
& \leq \sum_{n=0}^{\infty}\left(2^{n} r\right)^{-d-\lambda} \mu\left(B\left(x_{0}, 2^{n+1} r\right)\right) \\
& \leq c r^{-d-\lambda} \sum_{n=0}^{\infty} 2^{-n(d+\lambda)}\left(2^{n+1} r\right)^{d} \leq c r^{-\lambda}
\end{aligned}
$$

This proves (2). The estimate (3) follows in a similar way.

From now on we let $F \subset \mathbb{R}^{N}$ be a connected $d$-set, $d \in(0, N], N \geq 2$, and we let $\mu$ be its $d$-measure. We put $t_{0}=\infty$ when $r_{0}=\infty$ and $t_{0}=r_{0}^{d_{w}}$ otherwise (see Definition 2.1). We often refer to the general theory of Markov processes in the setting of $[\mathrm{BG}]$ or $[\mathrm{ChZ}]$.

Definition 2.1. A Markov process $X=\left(P^{x}, X_{t}\right)_{x \in F, t \geq 0}$ is called a fractional diffusion on $F$ if

(a) $X$ is a diffusion with state space $F$, 
(b) $X$ has a symmetric transition density $q(t, x, y)=q(t, y, x), t>0$, $x, y \in F$, which is jointly continuous for each $t>0$ and satisfies, for some constants $c_{1}, \ldots, c_{4}>0, d_{w}>1$ and all $x, y \in F$ and $t \in\left(0, t_{0}\right)$,

$$
\begin{aligned}
& c_{1} t^{-d_{s} / 2} \exp \left(-c_{2}\left(\frac{|x-y|}{t^{1 / d_{w}}}\right)^{\gamma}\right) \\
& \quad \leq q(t, x, y) \leq c_{3} t^{-d_{s} / 2} \exp \left(-c_{4}\left(\frac{|x-y|}{t^{1 / d_{w}}}\right)^{\gamma}\right) .
\end{aligned}
$$

Here $d_{s}=2 d / d_{w}, \gamma=d_{w} /\left(d_{w}-1\right)$.

It is also known (see $[\mathrm{P}]$ ) that all fractional diffusions on a fixed $d$-set $F$ must have the same value of the constant $d_{w}$, i.e. $d_{w}$ depends only on the underlying geometry. We have $d_{w}=2$ for $F=\mathbb{R}^{N}$ and if the heat kernel of the diffusion satisfies (4) then $d_{w} \geq 2$ (see [G]). We note here that the above definition differs from that given in $[\mathrm{Ba}]$. Following $[\mathrm{P}]$ we use the Euclidean distance instead of the intrinsic shortest path metric (see [Ba]). Since the well known fractal diffusions were constructed in the shortest path metric setting (e.g. $[\mathrm{Ba}]$ ), (4) is virtually tantamount to the assumption that the two metrics are equivalent.

3. Stable process. From now on we fix $\alpha \in(0,2)$. We also assume that $F$ is a connected $d$-set with $d$-measure $\mu$ and $r_{0}=\infty$ in (1). In particular $d \geq 1$ and $F$ is necessarily unbounded. We briefly recall the construction of the $\alpha$-stable process from $[\mathrm{S}]$. Suppose that there exists a fractional diffusion on $F$ and let $q(u, x, y), u>0, x, y \in F$, denote its transition density with respect to $\mu$. Let $\left(Y_{t}\right)_{t>0}$ be the $\alpha / 2$-stable subordinator given by the Laplace transform $E \exp \left(-u Y_{t}\right)=\exp \left(-t u^{\alpha / 2}\right)$. Let $\eta_{t}(u), t>0, u \geq 0$, be its onedimensional distribution density (see $[\mathrm{Be}]$ or $[\mathrm{BG}]$ for more details). For $t>0$ and $x, y \in F$ we define

$$
p(t, x, y)=\int_{0}^{\infty} q(u, x, y) \eta_{t}(u) d u .
$$

By the general theory $p(t, x, y)$ is the transition density of a Markov process called the subordinate process (see [BG, p. 18]), which we denote by $\left(X_{t}\right)_{t>0}$ and call $\alpha$-stable.

To simplify the notation, for the rest of the paper we let $d_{\alpha}=d+\alpha d_{w} / 2$. The main result of this section is the theorem below. It resembles a well known estimate for the rotation invariant $\alpha$-stable process on $\mathbb{R}^{N}$ and can be interpreted as weak scaling of our process.

Theorem 3.1 (Weak scaling). For $t>0, x, y \in F, x \neq y$, we have

$$
p(t, x, y) \asymp \min \left(\frac{t}{|x-y|^{d_{\alpha}}}, t^{-d_{s} / \alpha}\right),
$$


in particular

(6)

$$
c_{1} t^{-d_{s} / \alpha} \leq p(t, x, x) \leq c_{2} t^{-d_{s} / \alpha} .
$$

Proof. By Theorem 37.1 of [D],

$$
\lim _{u \rightarrow \infty} \eta_{1}(u) u^{1+\alpha / 2}=\alpha /(2 \Gamma(1-\alpha / 2)) .
$$

This, boundedness of $\eta_{1}(\cdot)$ and the scaling property

$$
\eta_{t}(u)=t^{-2 / \alpha} \eta_{1}\left(t^{-2 / \alpha} u\right), \quad t, u>0,
$$

yield the following estimates:

$$
\begin{array}{ll}
\eta_{t}(u) \leq c_{3} t u^{-1-\alpha / 2}, & t, u>0, \\
\eta_{t}(u) \geq c_{4} t u^{-1-\alpha / 2}, & t>0, u>u_{0} t^{2 / \alpha},
\end{array}
$$

where $u_{0}$ depends only on $\alpha$. Let $t>0, x, y \in F, x \neq y$, and $d(t, x, y)=$ $|x-y|^{\gamma} t^{-2 /\left(\alpha\left(d_{w}-1\right)\right)}$. By the definition of $p(t, x, y)$, (10) and substitution $v=c_{2}|x-y|^{\gamma} u^{-1 /\left(d_{w}-1\right)}$ (where $c_{2}$ is defined in (4)) we get

$$
\begin{aligned}
p(t, x, y) & \geq c t \int_{u_{0} t^{2 / \alpha}}^{\infty} u^{-d_{s} / 2} \exp \left(-c_{2}|x-y|^{\gamma} u^{-1 /\left(d_{w}-1\right)}\right) u^{-1-\alpha / 2} d u \\
& \geq c t|x-y|^{-d_{\alpha}} \int_{0}^{c_{5} d(t, x, y)} v^{\left(d_{s}+\alpha\right)\left(d_{w}-1\right) / 2-1} e^{-v} d v \\
& \geq c t|x-y|^{-d_{\alpha}} e^{-c_{5} d(t, x, y)} \int_{0}^{c_{5} d(t, x, y)} v^{\left(d_{s}+\alpha\right)\left(d_{w}-1\right) / 2-1} d v \\
& =c t^{-d_{s} / \alpha} e^{-c_{5} d(t, x, y)} .
\end{aligned}
$$

If $t \geq|x-y|^{\alpha d_{w} / 2}$ or $|x-y| t^{-2 /\left(\alpha d_{w}\right)} \leq 1$, then $d(t, x, y) \leq 1$ and $\exp \left(-c_{5} d(t, x, y)\right) \geq \exp \left(-c_{5}\right)$. Consequently, we then get

$$
p(t, x, y) \geq c_{6} t^{-d_{s} / \alpha} \text {. }
$$

Thus

$$
p(t, x, y) \geq c_{6} \min \left(\frac{t}{|x-y|^{d_{\alpha}}}, t^{-d_{s} / \alpha}\right)
$$

in this case. On the other hand, if $t<|x-y|^{\alpha d_{w} / 2}$ then $d(t, x, y)>1$ so that the integral in (11) is bounded away from 0 and $p(t, x, y) \geq c_{7} t|x-y|^{-d_{\alpha}} \geq$ $c_{7} \min \left(t /|x-y|^{d_{\alpha}}, t^{-d_{s} / \alpha}\right)$ again. By (4), (9) and the same substitution as in $(11)$,

$$
\begin{aligned}
p(t, x, y) & \leq c_{5} t \int_{0}^{\infty} u^{-d_{s} / 2-\alpha / 2-1} \exp \left(-c_{6}|x-y|^{\gamma} u^{-1 /\left(d_{w}-1\right)}\right) d u \\
& =\frac{c t}{|x-y|^{d_{\alpha}}} \Gamma\left(\left(d_{s}+\alpha\right)\left(d_{w}-1\right) / 2\right)
\end{aligned}
$$


This gives the upper bound of (5) with the first term under the minimum.

To finish the proof of (5) we will verify the estimate

$$
\eta_{t}(u) \leq c t u^{-1-\alpha / 2} \exp \left(-t u^{-\alpha / 2}\right), \quad u>0, t>0 .
$$

Indeed, from Lemma 1 in $[\mathrm{H}]$ (see also the proof therein) we have

$$
\eta_{1}(u) \leq c u^{(\alpha-4) /(4-2 \alpha)} \exp \left(-c_{8} u^{-\alpha /(2-\alpha)}\right)
$$

as $u \rightarrow 0$. Since $\alpha /(2-\alpha)>\alpha / 2$, we clearly have

$$
u^{(\alpha-4) /(4-2 \alpha)} e^{-c_{8} u^{-\alpha /(2-\alpha)}}=o\left(u^{-1-\alpha / 2} e^{-u^{-\alpha / 2}}\right) \quad \text { as } u \rightarrow 0 .
$$

From this and (9),

$$
\eta_{1}(u) \leq c u^{-1-\alpha / 2} \exp \left(-u^{-\alpha / 2}\right), \quad u>0 .
$$

(14) follows from this and (8). By (14) and the substitution $v=t u^{-\alpha / 2}$, for any $x, y \in F$ we obtain

$$
\begin{aligned}
p(t, x, y) & \leq c t \int_{0}^{\infty} u^{-d_{s} / 2} u^{-1-\alpha / 2} \exp \left(-t u^{-\alpha / 2}\right) d u \\
& =c t^{-d_{s} / \alpha} \int_{0}^{\infty} v^{d_{s} / \alpha} e^{-v} d v=c t^{-d_{s} / \alpha} .
\end{aligned}
$$

This completes the proof of (5) and also gives the upper bound in (6). The lower bound in (6) follows from (5) by continuity; see Proposition 3.2 below.

REMARK 1. Note that

$$
t \leq|x-y|^{\alpha d_{w} / 2} \text { if and only if } \frac{t}{|x-y|^{d_{\alpha}}} \leq t^{-d_{s} / \alpha} .
$$

Therefore, Theorem 3.1 can be reformulated in the following way:

$$
p(t, x, y) \asymp t^{-d_{s} / \alpha} \varphi\left(|x-y| t^{-2 /\left(\alpha d_{w}\right)}\right), \quad t>0, x, y \in F,
$$

with $\varphi(u)=(1 \vee|u|)^{-d_{\alpha}} \asymp(1+|u|)^{-d_{\alpha}}$.

REMARK 2. For later convenience we note that given $\beta \in(0,1)$ and $t \geq \beta|x-y|^{\alpha d_{w} / 2}$, the inequality (12) still holds true with some $c_{6}=c_{6}(\beta)$.

Consider a Markov process with state space being an open set $D \subseteq F$ and transition probability semigroup $P_{t}(x, E), t>0, x \in D$, where $E$ is a Borel subset of $D$. We say that the semigroup has the strong Feller property if $P_{t}\left(L^{\infty}(D, \mu)\right) \subseteq C_{\mathrm{b}}(D), t>0$, where $C_{\mathrm{b}}(D)$ stands for the continuous bounded functions on $D$. By $C_{0}(D)$ we denote the set of continuous bounded functions on $D$ that tend to zero at the boundary (and also when $x \rightarrow \infty$ if $D$ is unbounded). The semigroup is said to be strongly continuous on a function space $S$ if

$$
\lim _{t \rightarrow 0}\left\|P_{t} f-f\right\|=0, \quad f \in S,
$$


where the norm is taken in $S$. In what follows, $S$ will be equal to $C_{0}(D)$ or $L^{p}(D), p \in[1, \infty)$. The semigroup is said to have the Feller property if $P_{t}\left(C_{0}(D)\right) \subseteq C_{0}(D)$ and $P_{t}$ is strongly continuous on $C_{0}(D)$.

We now return to the study of our stable process on $F$.

Proposition 3.2. (i) The transition density $p(t, x, y)$ is jointly continuous in $(t, x, y) \in(0, \infty) \times F \times F$ for each $t>0$.

(ii) The transition semigroup $\left(P_{t}\right)$ generated by our stable process has both the Feller and strong Feller properties. In particular, $\left(P_{t}\right)$ is strongly continuous on $C_{0}(F)$.

Proof. One applies the same arguments as for the Brownian motion on $\mathbb{R}^{N}$ using properties of the underlying fractional diffusion and the dominated convergence theorem together with the upper bounds from (5), (6) and (14).

By virtue of (ii) in the above proposition we may and do assume that path functions of our stable process are right-continuous with left hand limits and that the process is quasi-left-continuous (see $[\mathrm{BG}]$ ).

4. Exit time. Let $E$ be a Borel subset of $F$. We let $T_{E}=\inf \{t \geq 0$ : $\left.X_{t} \in E\right\}$ and $\tau_{E}=T_{E^{c}}$.

Lemma 4.1. Let $\kappa>1$. There exist constants $c_{1}$ and $c_{2}$ such that for every $x \in F$ and $r>0, t>0$,

$$
P^{x}\left[X_{t} \notin B(x, r)\right] \geq c_{2} t r^{-\alpha d_{w} / 2}, \quad 0<t<r^{\alpha d_{w} / 2},
$$

and

$$
P^{y}\left[X_{t} \notin B(x, r)\right] \leq c_{1}(\kappa /(\kappa-1))^{\alpha d_{w} / 2} t r^{-\alpha d_{w} / 2},
$$

for all $y \in B(x, r / \kappa)$.

Proof. Let $a>1$ and let $x, r, t$ be as in (17). If $|y-x| \geq r$ then $|y-x|^{\alpha d_{w} / 2}$ $\geq t$, and by (5) and (16) we have

$$
\begin{aligned}
P^{x}\left[X_{t} \notin B(x, r)\right] & \geq \int_{r \leq|y-x|<a r} p(t, x, y) d \mu(y) \geq c t \int_{r \leq|y-x|<a r} \frac{d \mu(y)}{|y-x|^{d \alpha}} \\
& \geq c t r^{-d_{\alpha}} a^{-d_{\alpha}} \mu(B(x, a r) \backslash B(x, r)) \\
& \geq c t r^{-\alpha d_{w} / 2} a^{-d_{\alpha}}\left(c_{1} a^{d}-c_{2}\right),
\end{aligned}
$$

where the constants $c_{1}, c_{2}$ come from (1). We can choose $a$ large enough to make the last factor positive and (17) follows.

We now fix $\kappa>1, x \in F, t>0$, and $r>0$. Let $r_{0}=r(\kappa-1) / \kappa$. Observe that if $y \in B(x, r / \kappa)$ then $B\left(y, r_{0}\right) \subseteq B(x, r)$. By Theorem 3.1 and (2) we 
obtain

$$
\begin{aligned}
P^{y}\left[X_{t} \notin B(x, r)\right] & \leq P^{y}\left[X_{t} \notin B\left(y, r_{0}\right)\right]=\int_{B\left(y, r_{0}\right)^{\mathrm{c}}} p(t, y, z) d \mu(z) \\
& \leq c t \int_{B\left(y, r_{0}\right)^{\mathrm{c}}}|z-y|^{-d_{\alpha}} d \mu(z) \leq c t r_{0}^{-\alpha d_{w} / 2}
\end{aligned}
$$

and (18) follows.

The following simple lemma will be used without further mention (see [ChZ, Proposition 1.20]).

Lemma 4.2. Let $B$ be a Borel set in F. For each $t>0$ and $x \in F$ we have $P^{x}\left[\tau_{B}=t\right]=0$.

LEMMA 4.3. For each $\kappa>1$ there exists $c_{1}=c_{1}(\kappa)$ such that for $x \in F$, $r>0, y \in B(x, r / \kappa)$ we have

$$
P^{y}\left[\tau_{B(x, r)}<t\right] \leq c_{1} t r^{-\alpha d_{w} / 2} .
$$

Proof. Let $1<\kappa_{1}<\kappa$ and $\lambda>1$ be such that $\kappa_{1} \lambda=\kappa$ (e.g. $\kappa_{1}=\lambda$ $=\sqrt{\kappa})$. Define $T=\tau_{B(x, \lambda r)}$. For $y \in B\left(x, r / \kappa_{1}\right)$ we have

$$
\begin{aligned}
P^{y}[T<t] & =P^{y}\left[X_{t} \notin B(x, r) ; T<t\right]+P^{y}\left[X_{t} \in B(x, r) ; T<t\right] \\
& \leq P^{y}\left[X_{t} \notin B(x, r)\right]+P^{y}\left[X_{t} \in B(x, r) ; T<t\right]=A+B .
\end{aligned}
$$

By (18), we obtain $A \leq c_{2} t r^{-\alpha d_{w} / 2}$ with $c_{2}=c_{2}\left(\kappa_{1}\right)$.

By the strong Markov property we have

$$
B=E^{y}\left[\left.P^{X(T)}\left[X_{t-u} \in B(x, r)\right]\right|_{u=T} ; T<t\right] .
$$

We now estimate the integrand in (19):

$$
\begin{aligned}
\left.P^{X(T)}\left[X_{t-u} \in B(x, r)\right]\right|_{u=T} & \leq\left.\sup _{z \in B(x, \lambda r)^{\mathrm{c}}} P^{z}\left[X_{t-u} \in B(x, r)\right]\right|_{u=T} \\
& \leq \sup _{u \leq t} \sup _{z \in B(x, \lambda r)^{\mathrm{c}}} P^{z}\left[X_{u} \in B(x, r)\right] \\
& \leq \sup _{u \leq t} \sup _{z \in B(x, \lambda r)^{\mathrm{c}}} P^{z}\left[X_{u} \notin B(z,(\lambda-1) r)\right] \\
& \leq c_{3} t r^{-\alpha d_{w} / 2},
\end{aligned}
$$

where $c_{3}=c_{3}(\lambda)$. Consequently,

$$
P^{y}[T<t] \leq c_{4} t r^{-\alpha d_{w} / 2}, \quad y \in B\left(x, r / \kappa_{1}\right),
$$

where $c_{4}=c_{4}(\kappa)$. Apply (20) to $r / \lambda$ instead of $r$ and the assertion follows.

Proposition 4.4. There exists $c_{1}$ such that for $x \in F, r>0$,

$$
\sup _{y \in B(x, r)} E^{y} \tau_{B(x, r)} \leq c_{1} r^{\alpha d_{w} / 2},
$$


and for each $\kappa>1$ there exists a constant $c_{2}=c_{2}(\kappa)>0$ such that

$$
E^{y} \tau_{B(x, r)} \geq c_{2}(\kappa) r^{\alpha d_{w} / 2}, \quad x \in F, y \in B(x, r / \kappa) .
$$

Proof. For each $y \in B(x, r)$, from (5) we have

$$
\begin{aligned}
P^{y}\left[\tau_{B(x, r)}>t\right] & \leq P^{y}\left[X_{t} \in B(x, r)\right]=\int_{B(x, r)} p(t, y, z) d \mu(z) \\
& \leq c t^{-d_{s} / \alpha} \mu(B(x, r)) \leq c r^{d} t^{-d_{s} / \alpha} .
\end{aligned}
$$

Hence,

$$
P^{y}\left[\tau_{B(x, r)}>c_{3} r^{\alpha d_{w} / 2}\right] \leq c r^{d} c_{3}^{-d_{s} / \alpha}\left(r^{\alpha d_{w} / 2}\right)^{-d_{s} / \alpha}=1 / 2
$$

for a suitably chosen value of $c_{3}$. Let $t_{0}=c_{3} r^{\alpha d_{w} / 2}$. Then, by the Markov property, for $k=1,2, \ldots$ we have

$$
\begin{aligned}
P^{y}\left[\tau_{B(x, r)}>(k+1) t_{0}\right] & =P^{y}\left[\tau_{B(x, r)} \circ \theta_{t_{0}}>k t_{0}, \tau_{B(x, r)}>t_{0}\right] \\
& =E^{y}\left[P^{X\left(t_{0}\right)}\left[\tau_{B(x, r)}>k t_{0}\right] ; \tau_{B(x, r)}>t_{0}\right] \\
& \leq P^{y}\left[\tau_{B(x, r)}>t_{0}\right] \sup _{z \in B(x, r)} P^{z}\left[\tau_{B(x, r)}>k t_{0}\right]
\end{aligned}
$$

(here $\theta$ stands for the standard shift operator on the space of trajectories). By induction we get

$$
P^{y}\left[\tau_{B(x, r)}>k t_{0}\right] \leq(1 / 2)^{k}, \quad y \in B(x, r), k=0,1, \ldots
$$

Thus,

$$
E^{y} \tau_{B(x, r)}=\int_{0}^{\infty} P^{y}\left[\tau_{B(x, r)}>t\right] d t \leq \sum_{k=0}^{\infty} t_{0} P^{y}\left[\tau_{B(x, r)}>k t_{0}\right]=2 c_{3} r^{\alpha d_{w} / 2},
$$

which gives (21).

By Lemma 4.3 there exists $c_{4}=c_{4}(\kappa)$ such that

$$
P^{y}\left[\tau_{B(x, r)}<c_{4} r^{\alpha d_{w} / 2}\right] \leq 1 / 2, \quad y \in B(x, r / \kappa) .
$$

It follows that for $t_{0}=c_{4} r^{\alpha d_{w} / 2}$ we have

$$
E^{y} \tau_{B(x, r)} \geq t_{0} P^{y}\left[\tau_{B(x, r)}>t_{0}\right] \geq(1 / 2) c_{4} r^{\alpha d_{w} / 2}, \quad y \in B(x, r / \kappa) .
$$

The proof is complete.

5. Killed process and Green function. Let $D \subseteq F$ be an open set. By $\left(P_{t}^{D}\right)$ we denote the semigroup generated by the process killed on exiting $D$, that is (see $[\mathrm{BG}]$ ),

$$
P_{t}^{D} f(x)=E^{x}\left[f\left(X_{t}\right) ; t<\tau_{D}\right]
$$

for, e.g., nonnegative or bounded Borel functions $f$ on $F$.

The following proposition summarizes properties of $P_{t}^{D}$. 
Proposition 5.1. Let $D \subseteq F$ be an open bounded set.

(i) The semigroup $\left(P_{t}^{D}\right)$ has both Feller and strong Feller properties.

(ii) The semigroup is determined by a transition density $p^{D}(t, x, y)$, i.e. for any nonnegative or bounded Borel function $f$ on $F$ we have

$$
P_{t}^{D} f(x)=\int_{D} f(y) p^{D}(t, x, y) d \mu(y), \quad x \in F .
$$

Moreover, for each $t>0, p^{D}(t, x, y)$ is continuous in $(x, y) \in D \times D$ and

$$
\begin{aligned}
& p^{D}(t, x, y)=p^{D}(t, y, x), \quad x, y \in F \backslash \partial D, t>0, \\
& p^{D}(t, x, y)=p(t, x, y)-r^{D}(t, x, y), \quad x, y \in F, t>0,
\end{aligned}
$$

with $r^{D}(t, x, y)=E^{x}\left[p\left(t-\tau_{D}, X_{\tau_{D}}, y\right) ; t>\tau_{D}\right]$;

$$
\begin{aligned}
& p^{D}(t, x, y)>0, \quad x, y \in D, t>0 \\
& p^{D}(t, x, y)=\int_{F} p^{D}(s, x, z) p^{D}(t-s, z, y) d \mu(z), \quad t>s>0, x, y \in F \\
& p^{D}(t, x, y)=0, \quad x \in D^{\mathrm{c}} .
\end{aligned}
$$

Proof. The standard arguments that can be found e.g. in [ChZ] (see also $[\mathrm{Bs}])$ work also in the present setting with the exception of (24). We give a proof of (24) similar to but more direct than the one in [CS].

Let $K \subseteq D$ be a compact set and let $x, y \in K$. Define $\varrho=\operatorname{dist}\left(K, D^{\mathrm{c}}\right)$. By (5) and Lemma 4.3, we have

$$
\begin{aligned}
r^{D}(t, x, y) & =E^{x}\left[p\left(t-\tau_{D}, X_{\tau_{D}}, y\right) ; t>\tau_{D}\right] \\
& \leq c E^{x}\left[\left(t-\tau_{D}\right)\left|X_{\tau_{D}}-y\right|^{-d_{\alpha}} \mid ; t>\tau_{D}\right] \\
& \leq c t \varrho^{-d_{\alpha}} P^{x}\left[\tau_{D}<t\right] \leq c t \varrho^{-d_{\alpha}} P^{x}\left[\tau_{B(x, \varrho)}<t\right] \leq c t^{2} \varrho^{-d-\alpha d_{w}} .
\end{aligned}
$$

Also, by Theorem 3.1,

$$
p(t, x, y)^{-1} \leq c \max \left(t^{d_{s} / \alpha}, t^{-1}|x-y|^{d_{\alpha}}\right),
$$

so that

$$
\frac{r^{D}(t, x, y)}{p(t, x, y)} \leq c \max \left(t^{2+d_{s} / \alpha}, t|x-y|^{d_{\alpha}}\right) \varrho^{-d-\alpha d_{w}} \leq c_{1} \max \left(t^{2+d_{s} / \alpha}, t\right),
$$

where $c_{1}=c_{1}(D, K)$. It follows from (23) that for $t<t_{0}=t_{0}(K)$ and $x, y \in K$,

$$
p^{D}(t, x, y)>\frac{1}{2} p(t, x, y)>0 .
$$

Fix arbitrary $x_{0}, y_{0} \in D$. Let $r>0$ be such that $K=\overline{B\left(x_{0}, r\right) \cup B\left(y_{0}, r\right)}$ $\subseteq D$. We then use $t_{0}=t_{0}(K)$ as above, $(26)$ and the semigroup property to obtain

$$
p^{D}(t, x, y) \geq \int_{K} p^{D}(t / 2, x, z) p^{D}(t / 2, z, y) d \mu(z)>0
$$


for $x, y \in K$ and $t<2 t_{0}$. Similarly, by induction

$$
p^{D}(t, x, y)>0, \quad x, y \in K, t<n t_{0}, n=1,2 \ldots,
$$

and (24) follows.

The estimate (27) below is taken from [R] (Lemma 6). For the reader's convenience we include a version of the proof.

LEMMA 5.2. We have

$$
p^{D}(t, x, y) \leq c t^{-1-d_{s} / \alpha} r^{\alpha d_{w} / 2}, \quad x, y \in F, t>0 .
$$

Proof. From the semigroup property (25) and the estimate (5) we have

$$
\begin{aligned}
p^{D}(t, x, y) & =\int_{D} p^{D}(t / 2, x, z) p^{D}(t / 2, z, y) d \mu(z) \\
& \leq \sup _{z \in D} p^{D}(t / 2, z, y) \int_{D} p^{D}(t / 2, x, z) d \mu(z) \\
& \leq c t^{-d_{s} / \alpha} P^{x}\left[\tau_{D}>t / 2\right] .
\end{aligned}
$$

Hence, by the elementary inequality

$$
P^{x}\left[\tau_{D}>t / 2\right] \leq 2 E^{x} \tau_{D} / t
$$

and (21) we get the assertion.

Let $D \subseteq F$ be an open set. We define the Green function of $D$ by

$$
G_{D}(x, y)=\int_{0}^{\infty} p^{D}(t, x, y) d t
$$

Proposition 5.1 implies that if $D$ is an open bounded set in $F$ then $G_{D}(x, y)$ has the following properties: it is symmetric and strictly positive on $D \times D$; it vanishes if $x \in D^{\mathrm{c}}$ or, by the symmetry of $p^{D}(t, x, y), y \in \operatorname{int}\left(D^{\mathrm{c}}\right)$. $G_{D}$ is extended continuous on $D \times D$ for $\alpha \leq d_{s}$ as can be verified by an adaptation of the corresponding proof from [ChZ] $\left(G_{D}(x, x)=\infty\right.$ by $\left.(26)\right)$. To show the continuity for $\alpha>d_{s}$ we note that by (6) and a version of (27),

$$
p^{D}(t, x, y) \leq c \min \left(t^{-d_{s} / \alpha}, t^{-1-d_{s} / \alpha}\right), \quad t>0, x, y \in F .
$$

Since this is integrable over $(0, \infty)$, the desired assertion follows from the bounded convergence theorem. We leave the details to the interested reader.

Here we give an expression for the potential kernel of the stable process $X_{t}$.

LEMMA 5.3. If $\alpha<d_{s}$ then

$$
K_{\alpha}(x, y):=\int_{0}^{\infty} p(t, x, y) d t \asymp|x-y|^{-d+\alpha d_{w} / 2}, \quad x, y \in F .
$$


Proof. By Theorem 3.1 and (16),

$$
\begin{aligned}
\int_{0}^{\infty} p(t, x, y) d t & \asymp|x-y|^{-d-\alpha d_{w} / 2} \int_{0}^{|x-y|^{\alpha d_{w} / 2}} t d t+\int_{|x-y|^{\alpha d_{w} / 2}}^{\infty} t^{-d_{s} / \alpha} d t \\
& \asymp|x-y|^{-d+\alpha d_{w} / 2} .
\end{aligned}
$$

Corollary 5.4. The process is transient if and only if $\alpha<d_{s}$.

Lemma 5.5. If $\alpha<d_{s}$ then for any open bounded $D \subseteq F$,

$$
G_{D}(x, y)=K_{\alpha}(x, y)-E^{x} K_{\alpha}\left(X_{\tau_{D}}, y\right)
$$

(unless $x=y \in D^{\mathrm{c}}$ ) and

$$
G_{D}(x, y) \leq K_{\alpha}(x, y) \asymp|x-y|^{-d+\alpha d_{w} / 2}, \quad x, y \in F .
$$

Proof. From (23) by a simple change of variable we obtain

$$
\begin{aligned}
G_{D}(x, y) & =\int_{0}^{\infty} p(t, x, y) d t-E^{x} \int_{\tau_{D}}^{\infty} p\left(t-\tau_{D}, X_{\tau_{D}}, y\right) d t \\
& =\int_{0}^{\infty} p(t, x, y) d t-E^{x} \int_{0}^{\infty} p\left(t, X_{\tau_{D}}, y\right) d t
\end{aligned}
$$

which is clearly (33).

Now, (34) follows immediately from (33) and Lemma 5.3.

Proposition 5.6. If $\alpha>d_{s}$ then

$$
G_{B(x, r)}(x, y) \leq c r^{-d+\alpha d_{w} / 2}, \quad x, y \in F, r>0 .
$$

Proof. Define $D=B(x, r)$. We have

$$
\int_{0}^{r^{\alpha d_{w} / 2}} p^{D}(t, x, y) d t \leq \int_{0}^{r^{\alpha d_{w} / 2}} p(t, x, y) d t \leq c \int_{0}^{r^{\alpha d_{w} / 2}} t^{-d_{s} / \alpha} d t .
$$

Since $\alpha>d_{s}$, we get

$$
\int_{0}^{r^{\alpha d_{w} / 2}} p^{D}(t, x, y) d t \leq c_{1} r^{-d+\alpha d_{w} / 2}, \quad x, y \in F .
$$

From (27) it follows that

$$
\int_{r^{\alpha d_{w} / 2}}^{\infty} p^{D}(t, x, y) d t \leq c r^{\alpha d_{w} / 2} \int_{r^{\alpha d_{w} / 2}}^{\infty} t^{-1-d_{s} / \alpha} d t=c r^{-d+\alpha d_{w} / 2} .
$$

Now, the assertion follows from (35), (36) and the definition of the Green function. 
Let $u$ be a Borel measurable function $u$ on $F$, which is bounded from below (above). We say that $u$ is $\alpha$-harmonic in an open set $U \subseteq F$ if

$$
u(x)=E^{x} u\left(X\left(\tau_{B}\right)\right), \quad x \in B,
$$

for every bounded open set $B$ with the closure $\bar{B}$ contained in $U$. We say that $u$ is regular $\alpha$-harmonic in $U$ if

$$
u(x)=E^{x} u\left(X\left(\tau_{U}\right)\right), \quad x \in U
$$

By the strong Markov property of $X$, regular $\alpha$-harmonic functions are $\alpha$ harmonic. We give an elementary proof of $\alpha$-harmonicity of $G_{D}(x, y)$.

Proposition 5.7. Let $D$ be an open bounded set in $F$ and $\alpha \neq d_{s}$. Then $G_{D}(\cdot, y)$ is $\alpha$-harmonic in $D \backslash\{y\}$ for any $y \in D$.

Proof. Fix $y \in D$ and let $U$ be an arbitrary open set with $\bar{U} \subseteq D \backslash\{y\}$. (In fact it is enough to assume $U \subseteq D$ and $\operatorname{dist}(U, y)>0$; we will use this later.) For $x \in U$ and a nonnegative Borel measurable function $\psi$ supported in $U^{\mathrm{c}}$ we have

$$
\begin{aligned}
E^{x} \int_{0}^{\tau_{D}} \psi\left(X_{t}\right) d t & =E^{x} \int_{0}^{\tau_{U}} \psi\left(X_{t}\right) d t+E^{x}\left[\int_{\tau_{U}}^{\tau_{D}} \psi\left(X_{t}\right) d t ; \tau_{U}<\tau_{D}\right] \\
& =E^{x}\left[E^{X_{\tau_{U}}} \int_{0}^{\tau_{D}} \psi\left(X_{t}\right) d t ; \tau_{U}<\tau_{D}\right],
\end{aligned}
$$

by the strong Markov property. In terms of the Green function of $D$ this is

$$
\begin{aligned}
\int_{D} G_{D}(x, z) \psi(z) d \mu(z) & =E^{x}\left[\int_{D} G_{D}\left(X_{\tau_{U}}, z\right) \psi(z) d \mu(z) ; \tau_{U}<\tau_{D}\right] \\
& =\int_{D} E^{x}\left[G_{D}\left(X_{\tau_{U}}, z\right) ; \tau_{U}<\tau_{D}\right] \psi(z) d \mu(z)
\end{aligned}
$$

For almost all $z \in D \cap U^{\mathrm{c}}$ (with respect to $\mu$ ) we obtain

$$
G_{D}(x, z)=E^{x}\left[G_{D}\left(X_{\tau_{U}}, z\right) ; \tau_{U}<\tau_{D}\right] .
$$

Now, let $\alpha>d_{s}$. The continuity of $G_{D}(x, \cdot)$ and boundedness over $D \cap U^{\mathrm{c}}$ (see Proposition 5.6) yield

$$
G_{D}(x, y)=E^{x}\left[G_{D}\left(X_{\tau_{U}}, y\right) ; \tau_{U}<\tau_{D}\right]
$$

On the set $\left\{\tau_{U}=\tau_{D}\right\}$ we have $X_{\tau_{U}} \in D^{\mathrm{c}}$ and hence $G_{D}\left(X_{\tau_{U}}, y\right)=0$. Hence, from (39) it follows that

$$
E^{x} G_{D}\left(X_{\tau_{U}}, y\right)=E^{x}\left[G_{D}\left(X_{\tau_{U}}, y\right) ; \tau_{U}<\tau_{D}\right]=G_{D}(x, y),
$$

which completes the proof for $\alpha>d_{s}$.

If $\alpha<d_{s}$ then $K_{\alpha}(\cdot, y)$ is $\alpha$-harmonic in $F \backslash\{y\}$. Indeed, by (33) applied to an open bounded set $V \subseteq F \backslash\{y\}$ with $\operatorname{dist}(y, V)>0$, we have

$$
E^{x} K_{\alpha}\left(X_{\tau_{V}}, y\right)=K_{\alpha}(x, y)-G_{V}(x, y) \text {. }
$$


Since $y \in \operatorname{int}\left(V^{\mathrm{c}}\right)$, we get $G_{V}(x, y)=0$ and consequently

$$
E^{x} K_{\alpha}\left(X_{\tau_{V}}, y\right)=K_{\alpha}(x, y),
$$

which gives our claim. Now, the assertion for $\alpha<d_{s}$ follows from (33) and the strong Markov property.

REMARK. It is also possible to derive (39) for $\alpha<d_{s}$ by modifying the method just applied for the case $\alpha>d_{s}$. Indeed, we take into account uniform integrability (see (34)) of the family $\left\{G_{D}(w, \widetilde{y}) \mathbf{1}_{B(y, \delta)}(w): \widetilde{y} \in\right.$ $B(y, \delta)\}$, where $\delta>0$ is such that $B(y, 2 \delta) \subseteq U^{\mathrm{c}}$, and estimates of the Poisson kernel for $U$ near $y$ similar to those given in Section 6 .

Lemma 5.8. There exist $a>1$ and $c$ such that for all $x, y \in F$,

$$
G_{D}(x, y) \geq c|x-y|^{-d+\alpha d_{w} / 2}
$$

where $D=B(x, a|x-y|)$.

Proof. Let $a>1$. Define $r=|x-y|$. By the definition of $G_{D}(x, y)$, Theorem 3.1 and (16) we obtain

$$
\begin{aligned}
G_{D}(x, y) & \geq \int_{0}^{|x-y|^{\alpha d_{w} / 2}}\left(p(t, x, y)-E^{x}\left[p\left(t-\tau_{D}, X_{\tau_{D}}, y\right) ; \tau_{D}<t\right]\right) d t \\
& \geq \int_{0}^{|x-y|^{\alpha d_{w} / 2}}\left(c_{1} t|x-y|^{-d_{\alpha}}-E^{x}\left[c_{2}\left(t-\tau_{D}\right)\left|X_{\tau_{D}}-y\right|^{-d_{\alpha}} ; \tau_{D}<t\right]\right) d t,
\end{aligned}
$$

where $c_{1}$ and $c_{2}$ are defined by the lower and upper bound in (5), respectively. Observe that $\left|X_{\tau_{D}}-y\right| \geq(a-1)|x-y|$. Now, let $a>1$ be such that $c_{2}(a-1)^{-d_{\alpha}} \leq c_{1} / 2$. It follows that

$$
\begin{aligned}
G_{D}(x, y) & \geq \int_{0}^{|x-y|^{\alpha d_{w} / 2}}\left(c_{1} t|x-y|^{-d_{\alpha}}-c_{2} t((a-1)|x-y|)^{-d_{\alpha}}\right) d t \\
& \geq\left(c_{1} / 2\right)|x-y|^{-d_{\alpha}} \int_{0}^{|x-y| d_{w} / 2} t d t=\left(c_{1} / 4\right)|x-y|^{-d+\alpha d_{w} / 2},
\end{aligned}
$$

which gives (41).

6. Harmonic measure. For $x, y \in F$ define

$$
N(x, y)=\lim _{t \rightarrow 0} \frac{p(t, x, y)}{t}=\lim _{t \rightarrow 0} \int_{0}^{\infty} q(u, x, y) \frac{\eta_{t}(u)}{t} d u .
$$

We claim that the limit exists everywhere and is finite off the diagonal. Indeed, from (7) and (8) we have

$$
\begin{aligned}
\lim _{t \rightarrow 0} t^{-1} \eta_{t}(u) & =\lim _{t \rightarrow 0} t^{-1-2 / \alpha} \eta_{1}\left(u t^{-2 / \alpha}\right)=u^{-1-\alpha / 2} \lim _{s \rightarrow \infty} s^{1+\alpha / 2} \eta_{1}(s) \\
& =[\alpha /(2 \Gamma(1-\alpha / 2))] u^{-1-\alpha / 2}, \quad u>0 .
\end{aligned}
$$


By (4) and (9), for $x \neq y$,

$t^{-1} q(u, x, y) \eta_{t}(u) \leq c_{2} u^{-d_{s} / 2} \exp \left(-c_{3}|x-y|^{\gamma} u^{-\gamma / d_{w}}\right) u^{-1-\alpha / 2}, \quad u \in(0, \infty)$.

Since this is integrable our claim follows by the dominated convergence theorem. For later use we note that

$$
N(x, y)=c_{1} \int_{0}^{\infty} q(u, x, y) u^{-1-\alpha / 2} d u,
$$

with $c_{1}=\alpha /(2 \Gamma(1-\alpha / 2))$.

Let $E$ be a Borel subset of $F$ and $x \in F$. Define

$$
n(x, E)=\int_{E} N(x, y) d \mu(y),
$$

Note that by Proposition 4.4, for a bounded Borel set $D \subseteq F$ we have

$$
\sup _{x \in F} E^{x} \tau_{D}<\infty
$$

We have the following formula (see $[\mathrm{IW}])$.

Proposition 6.1. Assume that $D \subseteq F$ is an open nonempty bounded set, $E \subseteq F$ is a Borel set and $\operatorname{dist}(E, D)>0$. Then

$$
P^{x}\left[X_{\tau_{D}} \in E\right]=\int_{D} G_{D}(x, y) n(y, E) d \mu(y)
$$

Proof. We need to check the following assumptions (A1) and (A2) from [IW]. Let $\mathbb{M}=\left(S, P^{x}, x \in S\right)$ be a Markov process on a locally compact, separable metric space $S$ which satisfies

(A1) The semigroup

$$
T_{t} f(x)=\int_{S} f(y) P(t, x, d y)
$$

maps $C_{0}(S)$ into $C_{0}(S)$ and is strongly continuous in $t \geq 0$.

(A2) There exists a positive kernel $n(x, E), x \in S, E \subseteq S$ a Borel subset, such that

$1^{\circ}$ If $\operatorname{dist}(x, E)>0$ then

$$
n(x, E)<\infty \text {. }
$$

$2^{\circ}$ For $f \in C_{0}(S)$ and a bounded open set $D$ with $\operatorname{dist}(D, \operatorname{supp} f)>0$,

$$
\sup _{x \in D, t>0} t^{-1} T_{t} f(x)<\infty
$$

and

$$
\lim _{t \rightarrow 0^{+}} t^{-1} T_{t} f(x)=\lim _{t \rightarrow 0^{+}} t^{-1} \int_{S} f(y) P(t, x, d y)=\int_{S} f(y) n(x, d y)
$$

for every $x \in D$. 
Assumption (A1) is satisfied by Proposition 3.2. To establish (A2), fix $D$ as above. Let $f \in C_{0}(D)$ be $\operatorname{such}$ that $\operatorname{dist}(D, \operatorname{supp} f) \geq \delta>0$. Then, by (5) and Lemma 2.1,

$$
\sup _{x \in D, t>0} t^{-1} P_{t} f(x) \leq c \sup _{x \in D} \int \frac{f(y) d \mu(y)}{|x-y|^{d_{\alpha}}} \leq c\|f\|_{\infty} \delta^{-d_{\alpha}}<\infty
$$

and

$$
\lim _{t \rightarrow 0} t^{-1} P_{t} f(x)=\lim _{t \rightarrow 0} t^{-1} \int f(y) p(t, x, y) d \mu(y)=\int f(y) n(x, d y),
$$

by bounded convergence.

Corollary 6.2. Under the assumptions of Proposition 6.1 we have

$$
P^{x}\left[X_{\tau_{D}} \in E\right] \asymp \int_{D E} \frac{G_{D}(x, y)}{|y-z|^{d_{\alpha}}} d \mu(z) d \mu(y) .
$$

Proof. By a substitution as in (11), from (43) for $x, y \in F, x \neq y$, it follows that

$$
\begin{aligned}
N(x, y) & \asymp \int_{0}^{\infty} u^{-1-\alpha / 2-d_{s} / 2} \exp \left(-c|x-y|^{\gamma} u^{-\gamma / d_{w}}\right) d u \\
& =c|x-y|^{-d_{\alpha}} \int_{0}^{\infty} v^{-\left(d_{w}-1\right)\left(-1-\alpha / 2-d_{s} / 2\right)-d_{w}} e^{-v} d v .
\end{aligned}
$$

This yields

$$
n(x, E) \asymp \int_{E} \frac{d \mu(y)}{|y-x|^{d_{\alpha}}}, \quad x \in F,
$$

and (45) follows from (44).

In particular, the above corollary implies that the distribution of $X_{\tau_{D}}$ is absolutely continuous with respect to $\mu$ on $\operatorname{int}\left(D^{\mathrm{c}}\right)$. The corresponding density (Poisson kernel) is denoted by $P_{D}(x, y)$.

Proposition 6.3. Let $D \subseteq F$ be an open bounded set and $\alpha \neq d_{s}$. Then the Poisson kernel $P_{D}(\cdot, \cdot)$ admits a version which is jointly continuous on $D \times \operatorname{int}\left(D^{\mathrm{c}}\right)$.

Proof. We claim that $N(x, y)$ is continuous on the set $S_{a}=\{(x, y) \in$ $F \times F:|x-y|>a\}$ for each $a>0$. Indeed, let $(x, y) \in S_{a}$ and $x_{n} \rightarrow x$ and $y_{n} \rightarrow y$ as $n \rightarrow \infty$. Then, for sufficiently large $n$, we have $\left(x_{n}, y_{n}\right) \in S_{a}$ and

$$
u^{-1-\alpha / 2} q\left(u, x_{n}, y_{n}\right) \leq c_{1} u^{-d_{s} / 2-1-\alpha / 2} \exp \left(-c_{2} a^{\gamma} u^{-\gamma / d_{w}}\right), \quad u \in(0, \infty) .
$$

Consequently, by (43) and dominated convergence we obtain

$$
N\left(x_{n}, y_{n}\right)=c_{3} \int_{0}^{\infty} q\left(u, x_{n}, y_{n}\right) u^{-1-\alpha / 2} d u \rightarrow c_{3} \int_{0}^{\infty} q(u, x, y) u^{-1-\alpha / 2} d u
$$

as $n \rightarrow \infty$. This shows our claim. 
We let

$$
P_{D}(x, y)=\int_{D} G_{D}(x, z) N(z, y) d \mu(z), \quad x \in D, y \in \operatorname{int}\left(D^{\mathrm{c}}\right) .
$$

Assume that $\alpha<d_{s}$. Fix $(x, y) \in D \times \operatorname{int}\left(D^{\mathrm{c}}\right)$ and let $x_{n} \rightarrow x, y_{n} \rightarrow y$. We may and do assume that $\operatorname{dist}\left(y_{n}, D\right) \geq \frac{1}{2} \operatorname{dist}(y, D), n=1,2 \ldots$ Then, by (34) and (46), for $\varepsilon>0$ such that $\varepsilon<\left(d_{s} / \alpha-1\right)^{-1}$ and for $R$ large enough we have

$$
\begin{aligned}
& \sup _{n} \int_{D}\left|G_{D}\left(x_{n}, z\right) N\left(z, y_{n}\right)\right|^{1+\varepsilon} d \mu(z) \\
& \leq c \sup _{n} \int_{D}\left|x_{n}-z\right|^{\left(-d+\alpha d_{w} / 2\right)(1+\varepsilon)}\left|z-y_{n}\right|^{-d_{\alpha}(1+\varepsilon)} d \mu(z) \\
& \leq c(\operatorname{dist}(y, D))^{-d_{\alpha}(1+\varepsilon)} \sup _{n} \int_{D}\left|x_{n}-z\right|^{\left(-d+\alpha d_{w} / 2\right)(1+\varepsilon)} d \mu(z) \\
& \leq c(\operatorname{dist}(y, D))^{-d_{\alpha}(1+\varepsilon)} \sup _{n} \int_{B\left(x_{n}, R\right)}\left|x_{n}-z\right|^{\left(-d+\alpha d_{w} / 2\right)(1+\varepsilon)} d \mu(z)<\infty
\end{aligned}
$$

by (3). It follows that $\left(G_{D}\left(x_{n}, \cdot\right) N\left(\cdot, y_{n}\right)\right)_{n \in \mathbb{N}}$ are uniformly integrable and consequently

$$
\begin{aligned}
\lim _{n \rightarrow \infty} P_{D}\left(x_{n}, y_{n}\right) & =\lim _{n \rightarrow \infty} \int_{D} G_{D}\left(x_{n}, z\right) N\left(z, y_{n}\right) d \mu(z) \\
& =\int_{D} G_{D}(x, z) N(z, y) d \mu(z)=P_{D}(x, y) .
\end{aligned}
$$

This completes the case $\alpha<d_{s}$. If $\alpha>d_{s}$ then the process is point-recurrent and the Green function for $D$ is bounded (see Proposition 5.6). Therefore, a similar but simpler argument applies.

Proposition 6.4. There is a constant c such that for each $\kappa>1, x_{0} \in F$, $r>0$ and for $c_{1}=((\kappa+1) /(\kappa-1))^{d_{\alpha}} c$ and $c_{2}=((\kappa-1) /(\kappa+1))^{d_{\alpha}} c$ we have

$$
\begin{array}{r}
P_{B\left(x_{0}, r\right)}(x, z) \leq c_{1} r^{\alpha d_{w} / 2}|x-z|^{-d_{\alpha}}, \quad x \in B\left(x_{0}, r\right), z \in B\left(x_{0}, \kappa r\right)^{\mathrm{c}}, \\
P_{B\left(x_{0}, r\right)}(x, z) \geq c_{2} r^{\alpha d_{w} / 2}|x-z|^{-d_{\alpha}}, \\
\quad x \in B\left(x_{0}, r / \kappa\right), z \in \operatorname{int}\left(B\left(x_{0}, r\right)^{\mathrm{c}}\right) .
\end{array}
$$

Proof. Let $x, y \in B=B\left(x_{0}, r\right)$. Then, for $z \in B\left(x_{0}, \kappa r\right)^{\mathrm{c}}$,

$$
|y-z| \geq\left|z-x_{0}\right|-\left|y-x_{0}\right| \geq \kappa r-r=(\kappa-1) r
$$

and consequently

$$
\begin{aligned}
|x-z| & \leq|y-z|+|y-x| \leq|y-z|+2 r \\
& \leq|y-z|+2|y-z| /(\kappa-1)=|y-z|(\kappa+1) /(\kappa-1) .
\end{aligned}
$$


Thus, from Proposition 4.4 it follows that

$$
\begin{aligned}
P_{B}(x, z) & \asymp \int_{B} G_{B}(x, y)|y-z|^{-d_{\alpha}} d \mu(y) \\
& \leq((\kappa+1) /(\kappa-1))^{d_{\alpha}}|x-z|^{-d_{\alpha}} E^{x} \tau_{B} \leq c_{1}|x-z|^{-d_{\alpha}} r^{\alpha d_{w} / 2},
\end{aligned}
$$

with $c_{1}=c_{1}(\kappa)$. This gives (49). Now, let $x \in B\left(x_{0}, r / \kappa\right), z \in \operatorname{int}\left(B\left(x_{0}, r\right)^{\mathrm{c}}\right)$. Then $|y-z| \leq|x-z|(\kappa+1) /(\kappa-1)$, and from (22) we obtain

$$
P_{B}(x, z) \geq c((\kappa-1) /(\kappa+1))^{d_{\alpha}}|x-z|^{-d_{\alpha}} E^{x} \tau_{B} \geq c_{2}|x-z|^{-d_{\alpha}} r^{\alpha d_{w} / 2},
$$

with $c_{2}=c_{2}(\kappa)$ (cf. (51)). This completes the proof.

LEMma 6.5. Let $\alpha<d_{s}$ and $D=B\left(x_{0}, r\right)$ where $x_{0} \in F$ and $r>0$ are arbitrary. Then for each $k \geq 2$ there exists a constant $c_{1}=c_{1}(k)$ such that

$$
P_{D}(x, y) \leq c_{1} r^{-d+\alpha d_{w} / 2} \delta(y)^{-\alpha d_{w} / 2}, \quad y \in B\left(x_{0}, k r\right) \cap D^{\mathrm{c}}, x \in B\left(x_{0}, r / 2\right),
$$
where $\delta(y)=\operatorname{dist}(y, D)$.

Proof. By (45) and (34) we obtain

$$
\begin{aligned}
P_{D}(x, y) & \asymp \int_{D} G_{D}(x, z)|z-y|^{-d_{\alpha}} d \mu(z) \\
& \leq c\left(\int_{B(x, r / 4)}+\int_{D \cap B(x, r / 4)^{\mathrm{c}}}\right)|x-z|^{-d+\alpha d_{w} / 2}|z-y|^{-d_{\alpha}} d \mu(z) .
\end{aligned}
$$

If $|x-z|<r / 4$ then $|z-y|>r / 4$, so that the first integral in (53) is not greater than

$$
\begin{aligned}
& c(r / 4)^{-d_{\alpha}} \int_{B(x, r / 4)}|x-z|^{-d+\alpha d_{w} / 2} d \mu(z) \\
& \quad \leq c(r / 4)^{-d_{\alpha}}(r / 4)^{\alpha d_{w} / 2}=c r^{-d+\alpha d_{w} / 2} r^{-\alpha d_{w} / 2} \leq c_{2} r^{-d+\alpha d_{w} / 2} \delta(y)^{-\alpha d_{w} / 2},
\end{aligned}
$$

by Lemma 2.1 and the fact that $\delta(y)<(k-1) r$. Here $c_{2}=c_{2}(k)$. The second integral in (53) does not exceed

$$
\begin{aligned}
& c r^{-d+\alpha d_{w} / 2} \int_{D \backslash B(x, r / 4)}|z-y|^{-d-\alpha d_{w} / 2} d \mu(z) \\
& \leq c r^{-d+\alpha d_{w} / 2} \int_{B(y, \delta(y))^{\mathrm{c}}}|z-y|^{-d-\alpha d_{w} / 2} d \mu(z) \leq c r^{-d+\alpha d_{w} / 2} \delta(y)^{-\alpha d_{w} / 2},
\end{aligned}
$$

by Lemma 2.1. This completes the proof.

7. Harnack inequality. Recall that $F \subseteq \mathbb{R}^{N}$. The main result of this section can be stated as follows.

Theorem 7.1 (Harnack inequality). Let $\alpha \in\left(0,2 / d_{w}\right) \cup\left(d_{s}, 2\right)$. Then there exist $c_{1}, c_{2}$ such that for any $x_{0} \in F, r>0$ and any function $h \geq 0$ 
(regular) $\alpha$-harmonic in $B\left(x_{0}, c_{1} r\right)$, we have

$$
h(x) \geq c_{2} h(y), \quad x, y \in B\left(x_{0}, r\right) .
$$

Since we can always take a smaller ball as the region of $\alpha$-harmonicity of our function, we may and do assume regular $\alpha$-harmonicity above. The method of proof depends on whether the process is point-recurrent or transient. Theorem 7.1 will be strengthened in Corollary 7.7 below.

7.1. Recurrent case. In this subsection we assume that $\alpha>d_{s}$, so that the process is point-recurrent (see Lemma 7.2 and Remark 3 below).

For $\lambda>0$ define the $\lambda$-potential

$$
G^{\lambda}(x, y)=\int_{0}^{\infty} e^{-\lambda t} p(t, x, y) d t, \quad x, y \in F .
$$

LEMMA 7.2. There exist $c_{1}$ such that

$$
G^{\lambda}(x, y) \leq c_{1} \lambda^{d_{s} / \alpha-1}, \quad x, y \in F .
$$

Proof. This follows immediately from the definition of $G^{\lambda}(x, y)$ and our basic estimate $p(t, x, y) \leq c t^{-d_{s} / \alpha}$ :

$$
G^{\lambda}(x, y) \leq c \int_{0}^{\infty} e^{-\lambda t} t^{-d_{s} / \alpha} d t=c \Gamma\left(1-d_{s} / \alpha\right) \lambda^{d_{s} / \alpha-1}
$$

REMARK 3. Since the $\lambda$-potential is bounded on $F \times F$, it follows that points have positive capacity and the process is point-recurrent (see e.g. [PS, Theorem 7.1]). In particular, $P^{x}\left[T_{\{y\}}<\infty\right]=1, x, y \in F$ (in fact, points are regular for themselves, see [BG, Ch. 6, Proposition 4.11]).

For simplicity we write $T_{y}:=T_{\{y\}}$.

LEMMA 7.3. There exist constants $a>1$ and $p_{0}$ such that

$$
P^{x}\left[T_{y}<\tau_{B(x, a|x-y|)}\right]>p_{0}, \quad x, y \in F .
$$

Proof. First, we prove a general fact:

$$
P^{x}\left[T_{y}<\tau_{D}\right]=G_{D}(x, y) / G_{D}(y, y) .
$$

Let $\delta>0$ be such that $B(y, \delta) \subseteq D$. From (39) with $U=D \backslash B(y, \delta)$ and $x \in U$ we obtain

$$
G_{D}(x, y)=E^{x}\left[G_{D}\left(X\left(\tau_{D \backslash B(y, \delta)}\right), y\right) ; T_{B(y, \delta)}<\tau_{D}\right] .
$$

Letting $\delta \rightarrow 0$, by the continuity and boundedness of $G_{D}(\cdot, y)$, we get $(56)$.

Now, let $a$ and $r=|x-y|$ be as in Lemma 5.8. Then, from (56) with $D=B(x, a|x-y|),(41)$ and Proposition 5.6, it follows that

$$
P^{x}\left[T_{y}<\tau_{D}\right] \geq c \frac{|x-y|^{-d+\alpha d_{w} / 2}}{(a r)^{-d+\alpha d_{w} / 2}} \geq p_{0},
$$

which completes the proof. 
Proof of Theorem 7.1 for $d_{s}<\alpha<2$. Let $a, p_{0}$ be the constants of Lemma 7.3 and $c_{1}$ be such that $B(x, 2 a r) \subseteq B\left(x_{0}, c_{1} r\right), x \in B\left(x_{0}, r\right)$. Then $B(x, a|x-y|) \subseteq B\left(x_{0}, c_{1} r\right)$ for any $x, y \in B\left(x_{0}, r\right)$. It follows that

$$
\begin{aligned}
h(x) & =E^{x} h\left(X_{\tau_{B(x, a|x-y|)}}\right) \geq E^{x}\left[h\left(X_{\tau_{B(x, a|x-y|)}}\right) ; T_{y}<\tau_{B(x, a|x-y|)}\right] \\
& =E^{x}\left[E^{X\left(T_{y}\right)}\left[h\left(X_{\tau_{B(x, a|x-y|)}}\right)\right] ; T_{y}<\tau_{B(x, a|x-y|)}\right] \\
& =h(y) P^{x}\left[T_{y}<\tau_{B(x, a|x-y|)}\right] \geq p_{0} h(y) .
\end{aligned}
$$

This proves Theorem 7.1 for $\alpha>d_{s}$.

7.2. Transient case. Throughout this subsection we consider the transient case $\alpha<d_{s}$ (see Corollary 5.4). However, due to our restricted knowledge on the Poisson kernel and some geometrical reasons, in the proof of the remaining part of Theorem 7.1 we also assume the more restrictive condition $\alpha<2 / d_{w}$. We say that $E$ is polar if $P^{x}\left[T_{E}<\infty\right]=0$ for all $x \in F$. For a Borel set $E$ let $\operatorname{dim}_{\mathrm{H}}(E)$ denote its Hausdorff dimension.

Lemma 7.4. Let $E$ be a Borel set in $F \subseteq \mathbb{R}^{N}$. If $\alpha<2\left(d-\operatorname{dim}_{\mathrm{H}}(E)\right) / d_{w}$ then $E$ is polar.

Proof. The proof is an application of some general facts from potential theory. For $t>0$ and a Borel measure $m$ with compact support in $\mathbb{R}^{N}$, such that $0<m\left(\mathbb{R}^{N}\right)<\infty$, let $\phi_{m, t}(x)$ be its $t$-potential:

$$
\phi_{m, t}(x)=\int \frac{d m(y)}{|x-y|^{t}} .
$$

For a compact set $K \subseteq \mathbb{R}^{N}$ define the $t$-capacity of $E$ by

$$
C_{t}(K)=\sup _{m}\left\{\left(\int \phi_{m, t}(x) d x\right)^{-1}\right\}
$$

where the supremum is taken over Borel measures $m$ such that supp $m \subseteq K$ and $m(K)=1$ (see [Fa1]). Equivalently (see [L]),

$$
C_{t}(K)=\sup \left\{m(K): \phi_{m, t} \leq 1\right\} .
$$

For an arbitrary $E \subseteq \mathbb{R}^{N}$ define

$$
C_{t}(E)=\sup \left\{C_{t}(K): K \text { is compact, } K \subseteq E\right\} .
$$

By Corollary 6.5 from [Fa1],

$$
\left.\operatorname{dim}_{\mathrm{H}}(E)=\inf \left\{t: C_{t}(E)=0\right)\right\}=\sup \left\{t: C_{t}(E)>0\right\} .
$$

When $\alpha d_{w} / 2<d$ (i.e. $\alpha<d_{s}$ ), by [BG, Ch. 6, Section 4], $E$ is polar if and only if $C_{d-\alpha d_{w} / 2}(E)=0$.

Now, by our assumption, $\operatorname{dim}_{\mathrm{H}}(E)<d-\alpha d_{w} / 2$ so that $C_{d-\alpha d_{w} / 2}(E)=0$. It follows that $E$ is polar, which is the assertion of the lemma. 
For $z \in B(0, r / 2)$ and $s \in(r, 2 r)$ let $K(z, s)=B(z(s-r) / r,(s+r) / 2)$. Observe that $B(0, r) \subseteq K(z, s) \subseteq B(0, s)$. Indeed, if $y \in B(0, r)$ then

$$
|y-z(s-r) / r| \leq|y|+|z|(s-r) / r \leq r+(s-r) / 2=(s+r) / 2,
$$

so $B(0, r) \subseteq K(z, s)$; in particular $F \cap K(z, s)$ is not empty $(0 \in F$, but $z(s-r) / r$ may belong to $\left.\mathbb{R}^{N} \backslash F\right)$. Also, if $y \in K(z, s)$ then

$$
|y| \leq|z|(s-r) / r+(s+r) / 2 \leq(s-r) / 2+(s+r) / 2=s,
$$

and consequently $K(z, s) \subseteq B(0, s)$. Note also that if $|z| \leq r / 2$ and $s_{1} \leq s_{2}$ then $K\left(z, s_{1}\right) \subseteq K\left(z, s_{2}\right)$. Indeed, if $x \in K\left(z, s_{1}\right)$ then

$$
\begin{aligned}
\left|x-z \frac{s_{2}-r}{r}\right| & \leq\left|x-z \frac{s_{1}-r}{r}\right|+|z|\left|\frac{s_{1}-r}{r}-\frac{s_{2}-r}{r}\right| \\
& \leq \frac{s_{1}+r}{2}+\frac{r}{2} \frac{s_{2}-s_{1}}{r}=\frac{s_{2}+r}{2},
\end{aligned}
$$

which means that $x \in K\left(z, s_{2}\right)$.

Lemma 7.5. Let $r>0$. There exists $z_{0} \in B(0, r / 12)$ (not necessarily in $F$ ) such that for almost all $s \in(r, 2 r)$ the stable process does not hit the boundary of $K\left(z_{0}, s\right)$ in $F$.

Proof. Let $s \in(r, 2 r)$ and $\partial K(z, s)=\partial B(z(s-r) / r,(s+r) / 2) \cap F$ (on the right hand side of this equality we consider the boundary of the ball in $\mathbb{R}^{N}$ ). From Theorem 8.1 and the Product Formula 7.3 in [Fa2] it follows that for almost all (with respect to the Lebesgue measure) $z \in B(0, r / 2) \subseteq \mathbb{R}^{N}$ we have $\operatorname{dim}_{\mathrm{H}}(\partial K(z, s)) \leq(N-1+d)-N=d-1$. Therefore, $\alpha<2 / d_{w} \leq$ $2\left(d-\operatorname{dim}_{\mathrm{H}}(\partial K(z, s))\right) / d_{w}$, and so $\partial K(z, s)$ is polar by Lemma 7.4. For $x \in F$ and a Borel set $A$ the mapping $(z, s) \mapsto P^{x}\left[X_{\tau_{K(z, s)}} \in A\right]$ is jointly measurable. We thus we obtain

$$
\int_{r}^{2 r} \int_{z \in B(0, r / 2)} P^{x}\left[X_{\tau_{K(z, s)}} \in \partial K(z, s)\right] d z d s=0 .
$$

Hence, by Fubini's theorem

$$
\int_{r}^{2 r} P^{x}\left[X_{\tau_{K(z, s)}} \in \partial K(z, s)\right] d s=0
$$

for almost all $z \in B(0, r / 2)$ and the assertion follows.

REMARK 4 . When the process does not hit the boundary of a region $D$, for every Borel $u \geq 0$,

$$
E^{x} u\left(X_{\tau_{D}}\right)=\int_{D^{\mathrm{c}}} P_{D}(x, y) u(y) d \mu(y) .
$$

This fact will be exploited in what follows. 
Proof of Theorem $\% .1$ for $\alpha \in\left(0,2 / d_{w}\right)$. Without losing generality we assume that $x_{0}=0$. Let $h$ be a positive function that is regular $\alpha$-harmonic in $B(0,2 r)$. We will show that $h(x) \leq \operatorname{ch}(\widetilde{x}), x, \widetilde{x} \in B(0, r / 2)$. Let

$$
\begin{aligned}
& h_{1}(x)=E^{x}\left[h\left(X_{\left.\tau_{B(0,2 r}\right)}\right) ; X_{\tau_{B(0,2 r)}} \in B(0,3 r)\right], \\
& h_{2}(x)=E^{x}\left[h \left(X_{\left.\left.\tau_{B(0,2 r)}\right) ; X_{\tau_{B(0,2 r)}} \in B(0,3 r)^{\mathrm{c}}\right] .}\right.\right.
\end{aligned}
$$

Then $h=h_{1}+h_{2}$ on $F$ and, by definition, the functions $h_{1}$ and $h_{2}$ are regular $\alpha$-harmonic in $B(0,2 r)$. Moreover, since supp $h_{2} \cap B(0,2 r)^{\mathrm{c}} \subseteq B(0,3 r)^{\mathrm{c}}$, for $x, \tilde{x} \in B(0, r / 2)$ we have, by Proposition 6.4 ,

$$
\begin{aligned}
h_{2}(x) & =\int_{B(0,3 r)^{\mathrm{c}}} h_{2}(y) P_{B(0,2 r)}(x, y) d \mu(y) \\
& \leq c r^{\alpha d_{w} / 2} \int_{B(0,3 r)^{\mathrm{c}}} h_{2}(y)|x-y|^{-d_{\alpha}} d \mu(y) \\
& \leq c r^{\alpha d_{w} / 2} \int_{B(0,3 r)^{\mathrm{c}}} h_{2}(y)|\widetilde{x}-y|^{-d_{\alpha}} d \mu(y) \\
& \leq c \int_{B(0,3 r)^{\mathrm{c}}} h_{2}(y) P_{B(0,2 r)}(\widetilde{x}, y) d \mu(y)=c h_{2}(\widetilde{x}) .
\end{aligned}
$$

Consequently, it is enough to show an analogous inequality for $h_{1}$. Since we do not know whether the process hits the boundary of $B(0,3 r / 4)$, we have the inequality

$$
h_{1}(\widetilde{x})=E^{\widetilde{x}} h_{1}\left(X_{\tau_{B(0,3 r / 4)}}\right) \geq \int_{B(0,3 r / 4)^{\mathrm{c}}} P_{B(0,3 r / 4)}(\widetilde{x}, y) h_{1}(y) d \mu(y) .
$$

Define $R=B(0,3 r) \backslash B(0, r)$. Note that $\operatorname{supp} h_{1} \subseteq B(0,3 r)$, and also, for $\widetilde{x} \in B(0, r / 2)$ and $y \in R$ we have $|\widetilde{x}-y| \leq 4 r$. From (58) and Proposition 6.4 it follows that

$$
\begin{aligned}
h_{1}(\widetilde{x}) & \geq \int_{R} P_{B(0,3 r / 4)}(\widetilde{x}, y) h_{1}(y) d \mu(y) \geq c r^{\alpha d_{w} / 2} \int_{R}|\widetilde{x}-y|^{-d_{\alpha}} h_{1}(y) d \mu(y) \\
& \geq c r^{-d} \int_{R} h_{1}(y) d \mu(y) .
\end{aligned}
$$

Set

$$
P(x, y)=\int_{r}^{2 r} P_{K\left(z_{0}, s\right)}(x, y) d s, \quad x \in B(0, r / 2), y \in R,
$$

with $z_{0}$ of Lemma 7.5 and the usual convention: $P_{D}(x, y)=0$ if $y \in D$. Then

$$
h_{1}(x)=r^{-1} \int_{R} h_{1}(y) P(x, y) d \mu(y), \quad x \in B(0, r / 2) .
$$

Indeed, by the fact that $\operatorname{supp} h_{1} \subseteq B(0,3 r), B(0, r) \subseteq K\left(z_{0}, s\right) \subseteq B(0,2 r)$, 
$\alpha$-harmonicity of $h_{1}$ on $B(0,2 r)$, Lemma 7.5 and Remark 4 we obtain

$$
\begin{aligned}
\int_{R} h_{1}(y) P(x, y) d \mu(y) & =\int_{R} h_{1}(y) \int_{r}^{2 r} P_{K\left(z_{0}, s\right)}(x, y) d s d \mu(y) \\
& =\int_{r}^{2 r} \int_{y \in R} h_{1}(y) P_{K\left(z_{0}, s\right)}(x, y) d \mu(y) d s \\
& =\int_{r}^{2 r} h_{1}(x) d s=r h_{1}(x),
\end{aligned}
$$

which gives our claim.

Next we prove that

$$
P(x, y) \leq c r^{1-d}, \quad x \in B(0, r / 2), y \in R .
$$

Define $\delta_{s}(y)=\operatorname{dist}\left(y, K\left(z_{0}, s\right)\right)$. Let $s_{0}(y)=\inf \left\{s>0: y \in K\left(z_{0}, s\right)\right\}$ so that $y \in K\left(z_{0}, s\right)$ if $s>s_{0}(y)$. Recall that $\left|z_{0}\right|<r / 12<r / 4$. Hence,

$$
B(0, r / 2) \subseteq B\left(\frac{z_{0}(s-r)}{r}, \frac{1}{2} \frac{s+r}{2}\right), \quad s \in(r, 2 r) .
$$

By Lemma 6.5 and the substitution $s=r(u+1)$, for $x \in B(0, r / 2)$ and $y \in R$ we get

$$
\begin{aligned}
P(x, y) & \leq c r^{-d+\alpha d_{w} / 2} \int_{r}^{2 r \wedge s_{0}(y)} \delta_{s}(y)^{-\alpha d_{w} / 2} d s \\
& \leq c r^{-d+\alpha d_{w} / 2} \int_{r}^{2 r \wedge s_{0}(y)}\left(\left|y-z_{0}(s-r) / r\right|-(s+r) / 2\right)^{-\alpha d_{w} / 2} d s \\
& =c r^{1-d+\alpha d_{w} / 2} \int_{0}^{1 \wedge u_{0}(y)}\left(\left|y-u z_{0}\right|-(u+2) r / 2\right)^{-\alpha d_{w} / 2} d u,
\end{aligned}
$$

where $u_{0}=u_{0}(y)=-1+s_{0}(y) / r \geq 0$. Moreover,

$$
\left|y-u z_{0}\right|-(u+2) r / 2=\frac{\left|y-u z_{0}\right|^{2}-(u+2)^{2} r^{2} / 4}{\left|y-u z_{0}\right|+(u+2) r / 2} \asymp \frac{1}{r} g(u),
$$

where $g$ is the numerator of the last fraction:

$$
g(u)=g_{y, z_{0}, r}(u)=u^{2}\left(\left|z_{0}\right|^{2}-r^{2} / 4\right)-u\left(2\left\langle z_{0}, y\right\rangle+r^{2}\right)+|y|^{2}-r^{2} .
$$

Observe that $g(0)>0$ and $\left|z_{0}\right|^{2}-r^{2} / 4<0$ and so $u=u_{0}$ is the unique nonnegative solution of $g(u)=0$. Hence

$$
\begin{aligned}
u_{0} & =\frac{2\left\langle z_{0}, y\right\rangle+r^{2}-\sqrt{\left(2\left\langle z_{0}, y\right\rangle+r^{2}\right)^{2}+\left(r^{2}-4\left|z_{0}\right|^{2}\right)\left(|y|^{2}-r^{2}\right)}}{2\left(\left|z_{0}\right|^{2}-r^{2} / 4\right)} \\
& =\frac{2\left(|y|^{2}-r^{2}\right)}{\sqrt{\left(2\left\langle z_{0}, y\right\rangle+r^{2}\right)^{2}+\left(r^{2}-4\left|z_{0}\right|^{2}\right)\left(|y|^{2}-r^{2}\right)}+2\left\langle z_{0}, y\right\rangle+r^{2}}
\end{aligned}
$$


Since $\left|z_{0}\right|<r / 12$, we have $2\left|\left\langle z_{0}, y\right\rangle\right| \leq 2\left|z_{0}\right| \cdot|y|<r^{2} / 2$ and $r^{2}-4\left|z_{0}\right|^{2} \asymp r^{2}$. Consequently, $u_{0} \asymp(|y|-r) / r$ and $g(0) / u_{0} \asymp r^{2}$. By concavity of $g(u)$ we have $g(u) \geq g(0)\left(1-u / u_{0}\right)$. From (60), (61) and the fact that $u_{0} \leq 2$ it follows that

$$
\begin{aligned}
P(x, y) & \leq c r^{1-d+\alpha d_{w} / 2} r^{\alpha d_{w} / 2} \int_{0}^{1 \wedge u_{0}} g(u)^{-\alpha d_{w} / 2} d u \\
& \leq c r^{1-d+\alpha d_{w}}\left(g(0) / u_{0}\right)^{-\alpha d_{w} / 2} \int_{0}^{1 \wedge u_{0}}\left(u_{0}-u\right)^{-\alpha d_{w} / 2} d u \\
& \leq c r^{1-d}\left[-\left(u_{0}-u\right)^{1-\alpha d_{w} / 2}\right]_{0}^{1 \wedge u_{0}} \leq c r^{1-d},
\end{aligned}
$$

which gives our claim. It follows that

$$
\begin{aligned}
h_{1}(x) & =r^{-1} \int_{R} h_{1}(y) P(x, y) d \mu(y) \leq c r^{-1} \int_{R} h_{1}(y) r^{1-d} d \mu(y) \\
& =c r^{-d} \int_{R} h_{1}(y) d \mu(y) \leq c h_{1}(\widetilde{x}) .
\end{aligned}
$$

Thus, for functions $\alpha$-harmonic in $B(0,2 r)$ we showed (54) except that $x, y \in$ $B\left(x_{0}, r\right)$ is replaced by $x, y \in B\left(x_{0}, r / 2\right)$. Hence, substituting $2 r$ for $r$ we get the assertion of Theorem 7.1 with $c_{1}=4$. The proof is complete.

The following lemma is motivated by [BBy]. It is a useful tool in the proof of the Boundary Harnack Principle and extends Theorem 7.1 slightly. For the reader's convenience we give the proof, as the general context is much different than the one in $[\mathrm{BBy}]$. We point out that the situation is completely different as compared to the diffusion case. This is due to the fact that the definition of $\alpha$-harmonic functions is a global one. In general, a ball in $F$ may be disconnected. These circumstances make the classical chain argument unavailable in our setting (compare also with Proposition 3.37 and Corollary 3.38 in [Ba]). A desired extension of (54) in the diffusion case is not even true, since on each component of a (disconnected) ball the harmonic function can be defined separately.

Lemma 7.6. Let $x_{1}, x_{2} \in F, r>0$ and $M \in \mathbb{R}$ satisfy $\left|x_{1}-x_{2}\right| \leq M r$. Let $u \geq 0$ be a function which is $\alpha$-harmonic in $B\left(x_{1}, r\right) \cup B\left(x_{2}, r\right)$. Then $\alpha$-harmonic

$$
c^{-1} M^{-d_{\alpha}} u\left(x_{2}\right) \leq u\left(x_{1}\right) \leq c M^{d_{\alpha}} u\left(x_{2}\right) .
$$

Proof. Without any loss of generality we assume $x_{1}=0$ and $\left|x_{2}\right| \geq 3 r / 2$, because otherwise we can consider smaller $r$. By Theorem 7.1 we obtain

$$
u(x) \geq c u\left(x_{2}\right), \quad x \in B\left(x_{2}, r / 4\right) .
$$


By (50), for $x \in B\left(x_{2}, r / 4\right)$ we have

$$
P_{B(0, r / 2)}(0, x) \geq c r^{\alpha d_{w} / 2}|x|^{-d_{\alpha}} \geq c r^{\alpha d_{w} / 2}(M r)^{-d_{\alpha}}=c M^{-d_{\alpha}} r^{-d} .
$$

It follows that

$$
u(0) \geq \int_{B\left(x_{2}, r / 4\right)} P_{B(0, r / 2)}(0, x) u(x) d \mu(x) \geq c \mu\left(B\left(x_{2}, r / 4\right)\right) M^{-d_{\alpha}} r^{-d} u\left(x_{2}\right),
$$

and, by (1) and symmetry, the assertion follows.

Corollary 7.7. Let $\alpha \in\left(0,2 / d_{w}\right) \cup\left(d_{s}, 2\right)$. For each $\kappa>1$ there exists $c_{1}=c_{1}(\kappa)$ such that for any $x_{0} \in F, r>0$ and any function $h \geq 0$ regular $\alpha$-harmonic in $B\left(x_{0}, r\right)$, we have

$$
h(x) \geq c_{1} h(y), \quad x, y \in B\left(x_{0}, r / \kappa\right) .
$$

Proof. As previously we assume $x_{0}=0$. It is enough to show

$$
c_{2} h(0) \leq h(x) \leq c_{3} h(0), \quad x \in B(0, r / \kappa),
$$

with $c_{2}, c_{3}=c(\kappa)$. Let $\widetilde{r}=(\kappa-1) r / \kappa$. Clearly, $B(x, \widetilde{r}) \subseteq B(0, r)$, so that $h$ is $\alpha$-harmonic in $B(x, \widetilde{r}) \cup B(0, \widetilde{r})$. From Lemma 7.6, with $\widetilde{r}$ and $M=1 /(\kappa-1)$, we obtain (63), and the assertion follows.

8. Estimates near boundary. Let $D$ be an open nonempty bounded subset of $F$. We will be interested in the decay rate of the exit time and $\alpha$-harmonic functions near the boundary of $D$. The main results of this section are Theorems 8.3 and 8.4.

To measure the regularity of a set we make the following definition. We say that $D \subseteq F$ has the outer fatness property if there are constants $c_{1}=c_{1}(D)$ and $R_{0}=R_{0}(D)$ such that

$$
\mu\left(D^{\mathrm{c}} \cap B(x, r)\right) \geq c_{1} r^{d}, \quad x \in \partial D, r \in\left(0, R_{0}\right) .
$$

It is clear that this holds for the interior of the natural cells and their finite unions in the Sierpiński gasket (see below for the definition). For the rest of this section we assume that $D$ satisfies (64).

In Lemma 8.1 through Theorem 8.4 for simplicity we assume that $\operatorname{diam}(D)=1$. We set $D_{n}=\left\{x \in D: \delta(x) \leq k^{-n}\right\}, n=0,1, \ldots$, where $\delta(x)=\operatorname{dist}\left(x, D^{\mathrm{c}}\right) \leq 1$ and $k$ is a natural number whose value will be specified later. Observe that $D_{0}=D$. Let $P_{n}=D_{n} \backslash D_{n+1}, n=0,1, \ldots$

LEMMA 8.1. There exists $c_{1}$ such that for each $a>2, r>0$ and $x \in F$,

$$
P^{x}\left[X_{\tau_{B(x, r)}} \notin B(x, a r)\right] \leq c_{1} a^{-\alpha d_{w} / 2} .
$$

Proof. Since $a>2$ we can apply Proposition 6.4 with $\kappa=2$ and get the constant independent of $a$. Thus 


$$
\begin{aligned}
P^{x}\left[X_{\tau_{B(x, r)}} \notin B(x, a r)\right] & \leq c r^{\alpha d_{w} / 2} \int_{B(x, a r)^{\mathrm{c}}}|x-y|^{-d_{\alpha}} d \mu(y) \\
& =c r^{\alpha d_{w} / 2} \sum_{m=0}^{\infty} \int_{a r 2^{m} \leq|y-x| \leq a r 2^{m+1}}|x-y|^{-d_{\alpha}} d \mu(y) \\
& \leq c r^{\alpha d_{w} / 2} \sum_{m=0}^{\infty} \mu\left(B\left(x, a r 2^{m+1}\right)\right)\left(a r 2^{m}\right)^{-d-\alpha d_{w} / 2} \\
& \leq c r^{\alpha d_{w} / 2}(a r)^{-\alpha d_{w} / 2} \sum_{m=0}^{\infty} 2^{-m \alpha d_{w} / 2}
\end{aligned}
$$

and the assertion follows.

REMARK. That the constant $c_{1}$ does not depend on $r$ and $a>2$ may be viewed as an instance of weak scaling for our process.

For the rest of this section let $B_{n}=B\left(x, k^{-n}\right)$.

Proposition 8.2. There exists $p=p(D)>0$ such that

$$
P^{x}\left[\tau_{D} \leq \tau_{B_{n}}\right] \geq p, \quad x \in D_{n} .
$$

Proof. We only need to prove the inequality for $n \geq 1$. Fix $x \in D_{n}$ and let $x_{0} \in D^{\mathrm{c}}$ be such that $\left|x-x_{0}\right| \leq k^{-n}$. Define $r_{n}=1 / k^{n}$ and $A_{n}=$ $B\left(x_{0}, r_{n}\right) \cap D^{\mathrm{c}}$. Then we have

$$
P^{x}\left[\tau_{D} \leq \tau_{B_{n}}\right] \geq P^{x}\left[X\left(\tau_{B_{n}}\right) \in D^{\mathrm{c}}\right] \geq \int_{A_{n}} P_{B\left(x, r_{n}\right)}(x, y) d \mu(y) .
$$

We choose $k$ large enough so that $1 / k<R_{0}$ in (64). Observe that if (64) holds for all points in $\partial D$ then, by (1), it holds for $x_{0}$ as well. Also, we have $|x-y| \leq\left|x-x_{0}\right|+\left|x_{0}-y\right| \leq 2 k^{-n}, y \in A_{n}$. Therefore, by (50), for $n \geq 1$ we have

$$
P^{x}\left[\tau_{D} \leq \tau_{B_{n}}\right] \geq c \int_{A_{n}} r_{n}^{\alpha d_{w} / 2}|x-y|^{-d_{\alpha}} d \mu(y) \geq c k^{n d} \mu\left(A_{n}\right) \geq c c_{1},
$$

where the constant $c_{1}$ comes from (64). This completes the proof.

THEOREM 8.3. There exist $\beta=\beta(D) \in(0,1)$ and $c_{0}=c_{0}(D)$ such that

$$
E^{x} \tau_{D} \leq c_{0} \delta(x)^{\beta}, \quad x \in D .
$$

Proof. Fix $x \in D$. Let $r_{n}$ be as in the proof of the preceding proposition. Define

$$
u_{n}=\sup \left\{E^{x} \tau_{D}: x \in D_{n}\right\} .
$$

Clearly, it is enough to show

$$
u_{n} \leq c_{3} c_{4}^{n}, \quad n=0,1, \ldots,
$$


with $c_{3}=c_{3}(D), c_{4}=c_{4}(D) \in(0,1)$. By the strong Markov property we have

$$
\begin{aligned}
E^{x} \tau_{D} & =E^{x}\left[\tau_{D} ; \tau_{B_{n}} \geq \tau_{D}\right]+E^{x}\left[\tau_{D} ; \tau_{B_{n}}<\tau_{D}\right] \\
& \leq E^{x} \tau_{B_{n}}+E^{x}\left[E^{X\left(\tau_{B_{n}}\right)} \tau_{D} ; \tau_{B_{n}}<\tau_{D}\right]
\end{aligned}
$$

By Proposition 4.4, the first term is not greater than $c r_{n}^{\alpha d_{w} / 2}=c k^{-n \alpha d_{w} / 2}$. The second one is equal to

$\sum_{j=0}^{n-2} E^{x}\left[E^{X\left(\tau_{B_{n}}\right)} \tau_{D} ; X\left(\tau_{B_{n}}\right) \in P_{j}\right]+E^{x}\left[E^{X\left(\tau_{B_{n}}\right)} \tau_{D} ; X\left(\tau_{B_{n}}\right) \in D_{n-1}\right]=A+B$

By Proposition 8.2,

$$
B \leq u_{n-1} P^{x}\left[\tau_{B_{n}} \leq \tau_{D}\right] \leq(1-p) u_{n-1} .
$$

Suppose that $n \geq 2$, for otherwise the term $A$ is absent. Then

$$
A \leq \sum_{j=0}^{n-2} u_{j} P^{x}\left[X\left(\tau_{B_{n}}\right) \in P_{j}\right]=\sum_{j=2}^{n} u_{n-j} P^{x}\left[X\left(\tau_{B_{n}}\right) \in P_{n-j}\right]
$$

Let $x_{0} \in D^{\mathrm{c}}$ be a point closest to $x$. Note that $|y-x| \leq 1 /\left(2 k^{n-j+1}\right)$ yields

$$
\delta(y) \leq\left|y-x_{0}\right| \leq|y-x|+\left|x-x_{0}\right| \leq 1 /\left(2 k^{n-j+1}\right)+1 / k^{n} \leq 1 / k^{n-j+1},
$$

provided $k \geq 2$ and $j \geq 2$. In other words, $P_{n-j} \subseteq B\left(x, r_{n} k^{j-1} / 2\right)^{\mathrm{c}}$. Consequently,

$$
\begin{aligned}
P^{x}\left[X\left(\tau_{B_{n}}\right) \in P_{n-j}\right] & \leq P^{x}\left[X\left(\tau_{B_{n}}\right) \notin B\left(x, r_{n} k^{j-1} / 2\right)\right] \\
& \leq c k^{-(j-1) \alpha d_{w} / 2} \leq c k^{-(j-1) \alpha d_{w} / 2}
\end{aligned}
$$

by Lemma 8.1 . We conclude that

$$
u_{n} \leq c_{1} k_{0}^{-n}+(1-p) u_{n-1}+c_{2} \sum_{j=2}^{n} u_{n-j} k_{0}^{-(j-1)}, \quad n=2,3, \ldots
$$

where $k_{0}=k^{\alpha d_{w} / 2}$. Let $c_{4}=c_{4}(D)$ be such that $1-p<c_{4}<1$. Fix any $n_{0} \geq 3$. We now choose the value of $k$ large enough to satisfy the following:

(a) $k_{0}^{1 / 4} c_{4}>1$

(b) $c_{2}<k_{0}^{1 / 4}$

(c) $\left(k_{0} c_{4}\right)^{-n_{0}}+(1-p) / c_{4}+\left(k_{0}^{1 / 4}-1\right)^{-1}<1$.

Moreover, we may and do choose $c_{3} \geq c_{1}$ such that for $m=0,1, \ldots, n_{0}$,

$$
u_{m} \leq c_{3} c_{4}^{m} \text {. }
$$

We now extend (66) to all $m$ by induction. Assume that (66) holds for $m=0,1, \ldots, n-1$. From (65) it follows that 


$$
\begin{aligned}
u_{n} & \leq c_{1} k_{0}^{-n}+(1-p) c_{3} c_{4}^{n-1}+c_{2} \sum_{j=2}^{n} c_{3} c_{4}^{n-j} k_{0}^{-j+1} \\
& \leq c_{3} c_{4}^{n}\left(1 /\left(c_{4} k_{0}\right)^{n}+(1-p) / c_{4}\right)+c_{2} c_{3} k_{0}^{-1 / 4} k_{0}^{5 / 4} \sum_{j=2}^{n} c_{4}^{n-j} k_{0}^{-j / 4} k_{0}^{-3 j / 4} \\
& \leq c_{3} c_{4}^{n}\left(1 /\left(c_{4} k_{0}\right)^{n_{0}}+(1-p) / c_{4}\right)+c_{3} c_{4}^{n} k_{0}^{5 / 4} \sum_{j=2}^{\infty} k_{0}^{-3 j / 4} \leq c_{3} c_{4}^{n},
\end{aligned}
$$

by our assumptions on $k_{0}$. This ends the proof.

The above method also applies to harmonic functions. Analogous results are used in $[\mathrm{B}],[\mathrm{JK}]$ in proofs of the Boundary Harnack Principle. In what follows we will assume the following inner fatness property of $D$.

There exist constants $\theta=\theta(D) \in(0,1)$ and $R_{0}=R_{0}(D)$ such that for every $r \in\left(0, R_{0}\right)$ and $Q \in \partial D$ there is a point $A=A(Q, r) \in D \cap B(Q, r)$ such that

$$
B(A, \theta r) \subseteq D \cap B(Q, r) .
$$

Since we can always take a smaller localization radius $R_{0}$, it is convenient to use the same symbol as in (64). It is clear that the interiors of a natural cell (or a finite sum of cells) in the Sierpiński gasket satisfy this condition (see below).

THEOREM 8.4. There exist constants $\beta=\beta(D), r_{0}=r_{0}(D)$ and $c_{0}=$ $c_{0}(D)$ such that for all $Q \in \partial D$ and $r \in\left(0, r_{0}\right)$, and functions $u \geq 0$ regular $\alpha$-harmonic in $D \cap B(Q, r)$ and satisfying $u(x)=0$ on $D^{\mathrm{c}} \cap B(Q, r)$, we have

$$
u(x) \leq c_{0}(|x-Q| / r)^{\beta} c(u), \quad x \in D \cap B(Q, r),
$$

where $c(u)=\sup \{u(y): y \in D \cap B(Q, r)\}$.

Proof. Let $D_{n}=D \cap B\left(Q, r / k^{n}\right), n=0,1, \ldots$, with $k \geq 2$ to be specified later. Define $u_{n}=\sup _{x \in D_{n}} u(x)$. We fix $n \geq 1$ and $x \in D_{n}$. Define $r_{n}=$ $r /\left(4 k^{n}\right)$ and $B_{n}=B\left(x, r_{n}\right)$. We have

$$
\begin{aligned}
u(x) & =E^{x} u\left(X\left(\tau_{D_{0}}\right)\right) \\
& =E^{x}\left[u\left(X\left(\tau_{D_{0}}\right)\right) ; \tau_{B_{n}}>\tau_{D_{0}}\right]+E^{x}\left[u\left(X\left(\tau_{D_{0}}\right)\right) ; \tau_{B_{n}} \leq \tau_{D_{0}}\right] .
\end{aligned}
$$

On the set $\left\{\tau_{B_{n}}>\tau_{D_{0}}\right\}$ we have

$$
X\left(\tau_{D_{0}}\right) \in D_{0}^{\mathrm{c}} \cap B_{n} \subseteq D_{0}^{\mathrm{c}} \cap B(Q, r)=D^{\mathrm{c}} \cap B(Q, r),
$$

so that $u\left(X\left(\tau_{D_{0}}\right)\right)=0$. Hence, by the strong Markov property

$$
\begin{aligned}
u(x) & =E^{x}\left[E^{X\left(\tau_{B_{n}}\right)} u\left(X\left(\tau_{D_{0}}\right)\right) ; \tau_{B_{n}} \leq \tau_{D_{0}}\right] \\
& \leq E^{x} u\left(X\left(\tau_{B_{n}}\right)\right) \\
& =E^{x}\left[u\left(X\left(\tau_{B_{n}}\right)\right) ; X\left(\tau_{B_{n}}\right) \notin B(Q, r)\right]+E^{x}\left[u\left(X\left(\tau_{B_{n}}\right)\right) ; X\left(\tau_{B_{n}}\right) \in B(Q, r)\right] .
\end{aligned}
$$


By our assumption, there is a ball $B(A, \theta r) \subseteq D \cap B(Q, r)$. Let $B_{A}=$ $B(A, \theta r / 2)$. Then

$$
\begin{aligned}
E^{x}\left[u\left(X\left(\tau_{B_{n}}\right)\right) ; X\left(\tau_{B_{n}}\right)\right. & \notin B(Q, r)] \\
& =\int_{B(Q, r)^{\mathrm{c}}} u(z) \frac{P_{B_{n}}(x, z)}{P_{B_{A}}(A, z)} P_{B_{A}}(A, z) d \mu(z) .
\end{aligned}
$$

For $x \in D_{n}$ and $z \in B(Q, r)^{\mathrm{c}}$ we have $|x-z| \geq r-r /\left(4 k^{n}\right) \geq r / 2 \geq 2 r_{n}$. Also $|A-z| \geq \theta r=2 r_{A}$, where $r_{A}=\theta r / 2$. It follows that we can apply Proposition 6.4, which gives

$$
\frac{P_{B_{n}}(x, z)}{P_{B_{A}}(A, z)} \leq c \frac{r_{n}^{\alpha d_{w} / 2}|x-z|^{-d_{\alpha}}}{r_{A}^{\alpha d_{w} / 2}|A-z|^{-d_{\alpha}}} \leq \frac{c}{k^{n \alpha d_{w} / 2}} \frac{|x-z|^{-d_{\alpha}}}{|A-z|^{-d_{\alpha}}} .
$$

If $|z-Q| \geq r$ then

$|x-z| \geq|z-Q|-|Q-x| \geq|z-Q|-r / k^{n} \geq|z-Q|\left(1-1 / k^{n}\right) \geq \frac{1}{2}|z-Q|$ and

$$
|A-z| \leq|z-Q|+|Q-A| \leq|z-Q|+r \leq 2|z-Q| .
$$

Combining this with (68) and (69) we obtain

$$
\begin{aligned}
E^{x}\left[u\left(X\left(\tau_{B_{n}}\right)\right) ; X\left(\tau_{B_{n}}\right) \notin B(Q, r)\right] & \leq c k^{-n \alpha d_{w} / 2} \int_{B(Q, r)^{\mathrm{c}}} u(z) P_{B_{A}}(A, z) d \mu(z) \\
& \leq c k^{-n \alpha d_{w} / 2} u(A) \leq c k^{-n \alpha d_{w} / 2} u_{0} .
\end{aligned}
$$

We need to estimate $E^{x}\left[u\left(X\left(\tau_{B_{n}}\right)\right) ; X\left(\tau_{B_{n}}\right) \in B(Q, r)\right]$. This can be done exactly as in the proof of Theorem 8.3. Consequently, we arrive at (65) and the same argument as before completes the proof.

Proposition 8.5 below is an analogue of the Carleson estimate. We adapt the proof from [BBy] (see also [B]). Our contribution is the control of the scale parameters in the computations. Since the process does not prefer any particular scale, the result cannot depend on it, and the weak scaling suffices to prove that independence.

Proposition 8.5. Let $\alpha<2 / d_{w}$. There exists a constant $c_{1}=c_{1}(\theta)$ such that for all $Q \in \partial D$ and $r \in\left(0, R_{0} / 2\right)$, and functions $u \geq 0$ regular $\alpha$-harmonic in $D \cap B(Q, 2 r)$ and satisfying $u(x)=0$ on $D^{\mathrm{c}} \cap B(Q, 2 r)$, we have

$$
u(x) \leq c_{1} u(A), \quad x \in D \cap B(Q, r),
$$

where $A$ is as in (67).

Proof. We assume $Q=0$. Let $K\left(z_{0}, s\right)$ be as in Lemma 7.5 and for $\sigma \in(r, 2 r)$ define

$$
u_{\sigma}(z)=E^{z} u\left(X\left(\tau_{K\left(z_{0}, \sigma\right)}\right)\right), \quad z \in F
$$


Then

$$
u(z) \leq u_{\sigma}(z), \quad z \in F
$$

Indeed,

$$
\begin{aligned}
u(z) & =E^{z} u\left(X\left(\tau_{D \cap K\left(z_{0}, \sigma\right)}\right)\right)=E^{z}\left[u\left(X\left(\tau_{D \cap K\left(z_{0}, \sigma\right)}\right)\right) ; X\left(\tau_{D \cap K\left(z_{0}, \sigma\right)}\right) \notin K\left(z_{0}, \sigma\right)\right] \\
& =E^{z}\left[u\left(X_{\left.\tau_{K\left(z_{0}, \sigma\right)}\right)}\right) ; X\left(\tau_{D \cap K\left(z_{0}, \sigma\right)}\right) \notin K\left(z_{0}, \sigma\right)\right] \\
& \leq E^{z} u\left(X_{\tau_{K\left(z_{0}, \sigma\right)}}\right)=u_{\sigma}(z) .
\end{aligned}
$$

We claim that there is $\sigma_{0}$ in $(7 r / 4,2 r)$ such that

$$
u_{\sigma_{0}}(0) \leq c r^{\alpha d_{w} / 2} \int_{|y|>r} u(y)|y|^{-d_{\alpha}} d \mu(y) .
$$

Indeed, by Lemma 7.5 and Remark 4 , the process does not hit the boundary of $K\left(z_{0}, \sigma\right)$ for almost all $\sigma \in(7 r / 4,2 r)$. Thus, we have

$$
\begin{aligned}
\int_{7 r / 4}^{2 r} u_{\sigma}(0) d \sigma & =\int_{7 r / 4}^{2 r} \int_{|y|>r} P_{K\left(z_{0}, \sigma\right)}(0, y) u(y) d \mu(y) d \sigma \\
& =\left(\int_{|y| \in(r, 4 r)}+\int_{|y|>4 r}\right) u(y) \int_{7 r / 4}^{2 r} P_{K\left(z_{0}, \sigma\right)}(0, y) d \sigma d \mu(y) \\
& =A+B .
\end{aligned}
$$

We estimate the integral $A$. Since $|y| \asymp r$, similarly to (59) we obtain

$$
\int_{7 r / 4}^{2 r} P_{K\left(z_{0}, \sigma\right)}(0, y) d \sigma \leq c r^{1-d} \asymp r^{1+\alpha d_{w} / 2}|y|^{-d_{\alpha}} .
$$

It follows that

$$
A \leq c r^{1+\alpha d_{w} / 2} \int_{|y| \in(r, 4 r)} u(y)|y|^{-d_{\alpha}} d \mu(y) .
$$

To estimate the integral $B$ in (72), observe that for $|y|>4 r$ we have $\left|y-z_{0}(s-r) / r\right|>3 r>2(\sigma+r) / 2$ and by Proposition 6.4 we get

$$
\int_{7 r / 4}^{2 r} P_{K\left(z_{0}, \sigma\right)}(0, y) d \sigma \leq c \int_{7 r / 4}^{2 r}((\sigma+r) / 2)^{\alpha d_{w} / 2}|y|^{-d_{\alpha}} d \sigma \leq c r^{1+\alpha d_{w} / 2}|y|^{-d_{\alpha}} \text {. }
$$

It follows that

$$
B \leq c r^{1+\alpha d_{w} / 2} \int_{|y|>4 r} u(y)|y|^{-d_{\alpha}} d \mu(y) .
$$

Consequently, we can estimate the mean value of $u_{\sigma}(0)$ over $\sigma \in(7 r / 4,2 r)$,

$$
(4 / r) \int_{7 r / 4}^{2 r} u_{\sigma}(0) d \sigma \leq c r^{\alpha d_{w} / 2} \int_{|y| \geq r} u(y)|y|^{-d \alpha} d \mu(y)
$$

which gives our claim (71). 
By the assumption (67), we fix a point $A$ such that $B(A, \theta r) \subseteq D \cap$ $B(Q, r)$. Since $u$ is regular $\alpha$-harmonic in $B(A, \theta r)$ by (50) we obtain

$$
\begin{aligned}
u(A) & \geq \int_{|y-A|>\theta r} P_{B(A, \theta r)}(A, y) u(y) d \mu(y) \\
& \geq c(\theta r)^{\alpha d_{w} / 2} \int_{|y-A|>\theta r}|y-A|^{-d_{\alpha}} u(y) d \mu(y) \\
& \geq c_{2} r^{\alpha d_{w} / 2} \int_{|y|>r}|y-A|^{-d_{\alpha}} u(y) d \mu(y),
\end{aligned}
$$

where $c_{2}=c_{2}(\theta)$. If $|y|>r$ then $|A|<r<|y|$, and consequently $|y-A| \leq$ $|y|+|A| \leq 2|y|$. Hence, by the above inequality and (71),

$$
u(A) \geq c c_{2} r^{\alpha d_{w} / 2} \int_{|y|>r}|y|^{-d_{\alpha}} u(y) d \mu(y) \geq c_{3} u_{\sigma_{0}}(0),
$$

with $c_{3}=c_{3}(\theta)$. From (70), Corollary 7.7 for the ball $B\left(0, \sigma_{0}\right)$ and $z \in$ $B(0, r)$, and (73) it follows that

$$
u(z) \leq u_{\sigma_{0}}(z) \leq c u_{\sigma_{0}}(0) \leq c_{4} u(A), \quad z \in B(0, r),
$$

where $c_{4}=c_{4}(\theta)$. This ends the proof.

8.1. Boundary Harnack Principle. Below we present a proof of the Boundary Harnack Principle for the Sierpiński gasket. For the sake of convenience, we recall here briefly the construction of the set (we introduce an unbounded version). Let $F_{0}$ be the closed convex triangle with vertices at $(0,0),(1,0)$ and $(1 / 2, \sqrt{3} / 2)$. Let $A$ be the interior of the triangle whose vertices are the midpoints of the edges of $F_{0}$. Let $F_{1}=F_{0} \backslash A$. Then $F_{1}$ consists of three closed triangles of sides $1 / 2$. To obtain $F_{2}$ we apply the above procedure to the triangles in $F_{1}$, and so on. Set

$$
F_{\infty}=\bigcap_{n=0}^{\infty} F_{n}, \quad F=\bigcup_{n=0}^{\infty} 2^{n} F_{\infty} .
$$

We call $F$ the (unbounded) Sierpinski gasket. The collection of those triangles in $\bigcup_{k=0}^{\infty} 2^{k} F_{n+k}$ (of sides $2^{-n}$ ) is denoted by $\mathcal{S}_{n}, n=0,1, \ldots$ Note that $F$ lies between the $x$-axis and the line $y=\sqrt{3} x$.

By a natural cell (or simply cell) we mean the intersection of $F$ with a triangle from $\mathcal{S}_{n}$ for some $n=0,1, \ldots$

We assume that our region $D$ is the interior of the sum of a finite number of natural cells (possibly of different sizes). In other words, there exist $n_{0} \in$ $\mathbb{N} \cup\{0\}, n_{i} \in \mathbb{N} \cup\{0\}, i=1, \ldots, n_{0}$, and $S_{i} \in \mathcal{S}_{n_{i}}$ such that

$$
D=\operatorname{int}\left(F \cap \bigcup_{i=1}^{n_{0}} S_{i}\right) \text {. }
$$


Note that the interior is taken with respect to the topology of $F$ (inherited from $\mathbb{R}^{N}$ ) and since $S_{i}$ are closed, any two adjacent cells always make a connected set. It is clear that (64) and (67) hold for our $D$ (with some $R_{0}=R_{0}(D)$ ). Moreover, observe that the distance between any two disjoint cells in $D$ is at least $R_{1}=R_{1}(D)>0$. We may and do assume that $R_{0}<R_{1}$. Since in the proof of the Boundary Harnack Principle we consider local neighborhoods of a boundary point, it is enough to deal with a single cell.

The result can be stated as follows.

Theorem 8.6 (Boundary Harnack Principle). Let $0<\alpha<2 / d_{w}$ or $d_{s}<\alpha<2$. There exists a constant $c_{1}=c_{1}(D)$ such that for all $Q \in \partial D$, $r \in\left(0, R_{0}\right)$, and functions $u, v \geq 0$ regular $\alpha$-harmonic in $D \cap B(Q, 2 r)$ which vanish on $D^{\mathrm{c}} \cap B(Q, 2 r)$ and satisfy $u\left(A_{1}\right)=v\left(A_{1}\right)$, where $A_{1}$ satisfying $(67)$ is defined below, we have

$$
c_{1}^{-1} v(x) \leq u(x) \leq c_{1} v(x), \quad x \in D \cap B(Q, r / 4) .
$$

Before the proof we make some clarifying remarks. Without losing generality we assume that our $d$-measure $\mu$ is the $d$-dimensional Hausdorff measure. As usual, we assume $Q=0$ and let $\Omega=D \cap B(Q, r)$. For arbitrary $r \in\left(0, R_{0}\right)$ we can always find $\widetilde{r}=2^{-n}$, for some $n \in \mathbb{N}$, such that $r / 2 \leq \widetilde{r}<r$. Thus, it is enough to prove (75) for $x \in D \cap B(Q, \widetilde{r} / 2)$. Therefore, we may and do assume that $r$ itself is of the form $r=2^{-n}$ with $n$ fixed. This implies that a ball with center at any vertex of any triangle from $\mathcal{S}_{n}$ and of radius $2^{-i} r, i \in \mathbb{N}$, consists of triangles from $\mathcal{S}_{n+i}$ (intersected with $F$ ). In particular, $\Omega$ is the intersection of $D$ with a triangle from $\mathcal{S}_{n}$ whose one vertex is at 0 (see Figure 1).

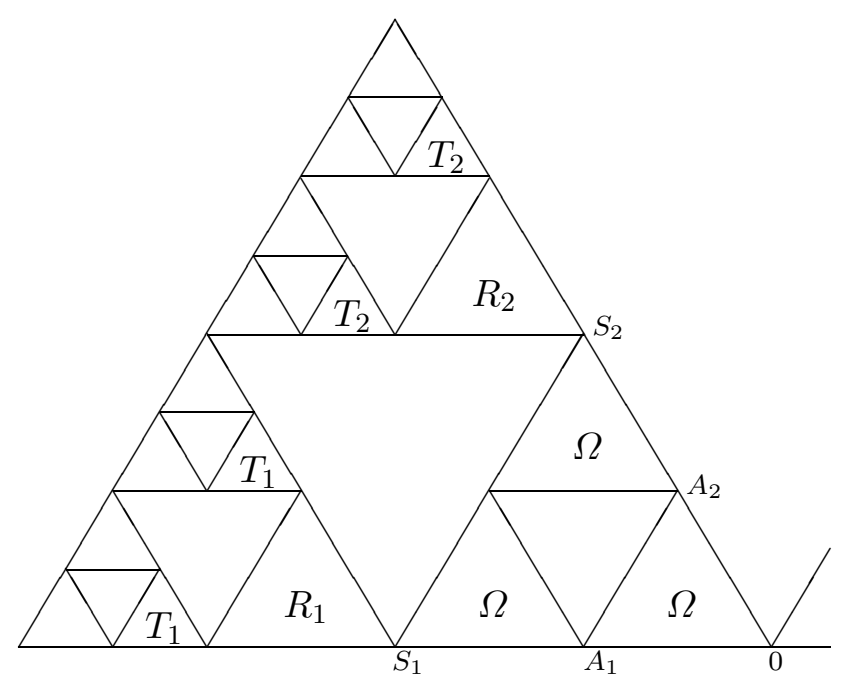

Fig. 1 
Let $\Omega_{1}=\frac{1}{2} \Omega=D \cap B(0, r / 2)$ and set $\left\{A_{1}, A_{2}\right\}=\partial \Omega_{1} \backslash\{0\}$ and $\left\{S_{1}, S_{2}\right\}=\partial \Omega \backslash\{0\}$, so that $S_{i}=2 A_{i}, i=1,2$. Define $R_{i}=B\left(S_{i}, r / 2\right) \cap \Omega^{\mathrm{c}}$, $i=1,2$, and $R=R_{1} \cup R_{2}$. Moreover, let $T_{i}=B\left(S_{i}, 3 r / 4\right) \backslash\left(\Omega \cup R_{i}\right), i=1,2$, and $T=T_{1} \cup T_{2}$.

Proof of the Boundary Harnack Principle. Let $u_{1}, u_{2}$ be functions such that

$$
u_{1}(y)=\left\{\begin{array}{ll}
u(y), & y \in R, \\
0, & y \in \Omega^{\mathrm{c}} \backslash R,
\end{array} \quad u_{2}(y)= \begin{cases}u(y), & y \in \Omega^{\mathrm{c}} \backslash R, \\
0, & y \in R,\end{cases}\right.
$$

and $u_{1}$ and $u_{2}$ are regular $\alpha$-harmonic in $\Omega$. Note that $u_{1}, u_{2} \geq 0$ and $u_{1}+u_{2}=u$. We define $v_{1}$ and $v_{2}$ analogously. By the Harnack inequality for $B\left(S_{i}, r\right), i=1,2$, we have

$$
u(y) \asymp u\left(A_{i}\right), \quad y \in R_{i} \cup T_{i}, i=1,2, \quad u\left(A_{2}\right) \asymp u\left(A_{1}\right) .
$$

Consequently,

$$
u(y) \asymp u\left(A_{1}\right), \quad y \in R \cup T .
$$

Fix $x \in \Omega$. From (76) it follows that

$$
u_{1}(x)=E^{x}\left[u\left(X_{\tau_{\Omega}}\right) ; X_{\tau_{\Omega}} \in R\right] \asymp u\left(A_{1}\right) P^{x}\left[X_{\tau_{\Omega}} \in R\right] .
$$

From the analogous relation for $v_{1}$ and our assumption $u\left(A_{1}\right)=v\left(A_{1}\right)$ we get

$$
u_{1}(x) \asymp v_{1}(x) \leq v(x), \quad x \in \Omega .
$$

Since $v_{2}=0$ on $R$ we have

$$
\begin{aligned}
v_{2}\left(A_{1}\right)= & E^{A_{1}} v_{2}\left(X_{\tau_{\Omega}}\right) \\
= & E^{A_{1}}\left[v\left(X_{\tau_{\Omega}}\right) ; X_{\tau_{\Omega}} \in T\right] \\
& +E^{A_{1}}\left[v\left(X_{\tau_{\Omega}}\right) ; X_{\tau_{\Omega}} \in(\Omega \cup R \cup T)^{\mathrm{c}}\right] .
\end{aligned}
$$

By the relation for $v$ analogous to $(76)$ we obtain

$$
\inf _{z \in T} v(z) \geq \inf _{z \in T \cup R} v(z) \asymp v\left(A_{1}\right) \asymp \sup _{z \in T \cup R} v(z),
$$

which yields

$$
E^{A_{1}}\left[v\left(X_{\tau_{\Omega}}\right) ; X_{\tau_{\Omega}} \in T\right] \geq c \sup _{z \in T \cup R} v(z) P^{A_{1}}\left[X_{\tau_{\Omega}} \in T\right] .
$$

On the other hand,

$$
\begin{aligned}
v\left(A_{1}\right) & =E^{A_{1}}\left[v\left(X_{\tau_{\Omega}}\right) ; X_{\tau_{\Omega}} \in R \cup T\right]+E^{A_{1}}\left[v\left(X_{\tau_{\Omega}}\right) ; X_{\tau_{\Omega}} \in(\Omega \cup R \cup T)^{\mathrm{c}}\right] \\
& \leq \sup _{z \in T \cup R} v(z) P^{A_{1}}\left[X_{\tau_{\Omega}} \in R \cup T\right]+E^{A_{1}}\left[v\left(X_{\tau_{\Omega}}\right) ; X_{\tau_{\Omega}} \in(\Omega \cup R \cup T)^{\mathrm{c}}\right] .
\end{aligned}
$$

Observe that

$$
P^{A_{1}}\left[X_{\tau_{\Omega}} \in R \cup T\right] \leq c_{0} P^{A_{1}}\left[X_{\tau_{\Omega}} \in T\right]
$$


Indeed, for $y \in T_{1}$ we have $\left|A_{1}-y\right| \leq 2 r$ and by (50) we obtain

$$
\begin{aligned}
P^{A_{1}}\left[X_{\tau_{\Omega}} \in T\right] & \geq P^{A_{1}}\left[X_{\tau_{B\left(A_{1}, r / 2\right)}} \in T_{1}\right] \geq c r^{\alpha d_{w} / 2} \int_{T_{1}}\left|A_{1}-y\right|^{-d_{\alpha}} d \mu(y) \\
& \geq c r^{\alpha d_{w} / 2}(2 r)^{-d_{\alpha}} \mu\left(T_{1}\right) \geq c_{0}^{-1} .
\end{aligned}
$$

Hence, we get

$$
\begin{aligned}
v\left(A_{1}\right) \leq & c \sup _{z \in T \cup R} v(z) P^{A_{1}}\left[X_{\tau_{\Omega}} \in T\right] \\
& +E^{A_{1}}\left[v\left(X_{\tau_{\Omega}}\right) ; X_{\tau_{\Omega}} \in(\Omega \cup R \cup T)^{\mathrm{c}}\right]
\end{aligned}
$$

Combining (78), (79) and (80) we get

$$
v_{2}\left(A_{1}\right) \geq c v\left(A_{1}\right) \text {. }
$$

Let $K=\Omega \cup R \cup\left(D^{\mathrm{c}} \cap B(0,2 r)\right)$. Observe that for $z \in \Omega$ and $y \in K^{\mathrm{c}}$ we have $|y-z| \asymp|y|$. Therefore, for $x \in \Omega$ we obtain

$$
\begin{aligned}
u_{2}(x) & =\int_{K^{\mathrm{c}}} P_{\Omega}(x, y) u(y) d \mu(y) \\
& \asymp \int_{K^{\mathrm{c}}}\left(\int_{\Omega} G_{\Omega}(x, z)|z-y|^{-d_{\alpha}} d \mu(z)\right) u(y) d \mu(y) \\
& \asymp \int_{K^{\mathrm{c}}}\left(\int_{\Omega} G_{\Omega}(x, z) d \mu(z)\right) u(y)|y|^{-d_{\alpha}} d \mu(y) \\
& =E^{x} \tau_{\Omega} \int_{K^{\mathrm{c}}} u(y)|y|^{-d_{\alpha}} d \mu(y)
\end{aligned}
$$

and the analogous relation for $v_{2}$. It follows that

$$
u_{2}(x) / u_{2}\left(A_{1}\right) \asymp E^{x} \tau_{\Omega} / E^{A_{1}} \tau_{\Omega} \asymp v_{2}(x) / v_{2}\left(A_{1}\right) .
$$

Denote the last quotient by $q_{0}$. Then, by (82), the definition of $u_{2}$, the assumption $u\left(A_{1}\right)=v\left(A_{1}\right)$ and (81),

$$
u_{2}(x) \leq c q_{0} u_{2}\left(A_{1}\right) \leq c q_{0} u\left(A_{1}\right)=c q_{0} v\left(A_{1}\right) \leq c q_{0} v_{2}\left(A_{1}\right)=c v_{2}(x), \quad x \in \Omega .
$$

Together with (77) and symmetry this completes the proof.

REMARK 5. We want to emphasize that in the particular case of the Sierpiński gasket, the Boundary Harnack Principle is a consequence of the Harnack inequality alone. It seems that a similar approach works for nested fractals. On the other hand the Boundary Harnack Principle for the Sierpiński carpet should be available by other, more complicated methods used in [B] for Lipschitz domains in $\mathbb{R}^{N}$.

Acknowledgements. The authors wish to thank T. Byczkowski, J. Dziubański and P. Graczyk for helpful discussions and comments. We are also grateful to an anonymous referee for useful comments and clarifying remarks. 
Note added in proof. After the paper was submitted we learned that Z.-Q. Chen and T. Kumagai [CK] studied general stable-like processes on fractals defined by means of appropriate Dirichlet forms. Their paper contains very interesting results, which partially overlap ours; however, their methods of proof are completely different, based on tightness results and the parabolic Harnack inequality.

\section{References}

[Ba] M. T. Barlow, Diffusions on fractals, in: Lectures on Probability Theory and Statistics, École d'Été de Probabilités de Saint-Flour XXV - 1995, Lecture Notes in Math. 1690, Springer, New York, 1998, 1-121.

[BB] M. T. Barlow and R. F. Bass, The construction of Brownian motion on the Sierpiński carpet, Ann. Inst. H. Poincaré Probab. Statist. 25 (1989), 225-257.

[BB1] - - - Brownian motion and harmonic analysis on Sierpiński carpets, Canad. J. Math. 51 (1999), 673-744.

[BP] M. T. Barlow and E. A. Perkins, Brownian motion on the Sierpiński gasket, Probab. Theory Related Fields 79 (1988), 543-623.

[Bs] R. F. Bass, Probabilistic Techniques in Analysis, Springer, New York, 1995.

[BL] R. F. Bass and D. A. Levin, Harnack inequalities for jump processes, Potential Anal. 17 (2002), 375-388.

[Be] J. Bertoin, Lévy Processes, Cambridge Univ. Press, Cambridge, 1996.

[BG] R. M. Blumenthal and R. K. Getoor, Markov Processes and Potential Theory, Pure Appl. Math. 29, Academic Press, New York, 1968.

[B] K. Bogdan, The boundary Harnack principle for the fractional Laplacian, Studia Math. 123 (1997), 43-80.

[BBy] K. Bogdan and T. Byczkowski, Probabilistic proof of boundary Harnack principle for $\alpha$-harmonic functions, Potential Anal. 11 (1999), 135-156.

[BSS] K. Bogdan, A. Stós and P. Sztonyk, Harnack inequality for symmetric stable processes on fractals, C. R. Acad. Sci. Paris Sér. I 335 (2002), 59-63.

[CK] Z.-Q. Chen and T. Kumagai, Heat kernel estimates for stable-like processes on d-sets, preprint, 2002.

[CS] Z.-Q. Chen and R. Song, Intrinsic ultracontractivity and conditional gauge for symmetric stable processes, J. Funct. Anal. 159 (1998), 267-294.

[ChZ] K. L. Chung and Z. Zhao, From Brownian Motion to Schrödinger's Equation, Springer, New York, 1995.

[DSV] K. Darlymple, R. Strichartz and J. P. Vinson, Fractal differential equations on the Sierpiński gasket, J. Fourier Anal. Appl. 5 (1999), 203-284.

[D] G. Doetsch, Introduction to the Theory and Application of the Laplace Transformation, Springer, New York, 1974.

[Fa1] K. Falconer, The Geometry of Fractal Sets, Cambridge Univ. Press, Cambridge, 1992.

[Fa2] K. Falconer, Fractal Geometry. Mathematical Foundations and Applications, Wiley, Chichester, 1990.

[FJ] W. Farkas and N. Jacob, Sobolev spaces on non-smooth domains and Dirichlet forms related to subordinate reflecting diffusions, Math. Nachr. 224 (2001), 75104.

[FOT] M. Fukushima, Y. Oshima and M. Takeda, Dirichlet Forms and Symmetric Markov Processes, de Gruyter, Berlin, 1994. 
[FU] M. Fukushima and T. Uemura, On Sobolev and capacitary inequalities for contractive Besov spaces over d-sets, Potential Anal. 18 (2003), 59-77.

[G] A. Grigor'yan, J. X. Hu and K.-S. Lau, Heat kernels on metric measure spaces and an application to semilinear elliptic equations, Trans. Amer. Math. Soc. 355 (2003), 2065-2095.

[H] J. Hawkes, A lower Lipschitz condition for the stable subordinator, Z. Wahrsch. Verw. Geb. 17 (1971), 23-32.

[HL] J. X. Hu and K.-S. Lau, Riesz potentials and Laplacians on fractals, preprint.

[IW] N. Ikeda and S. Watanabe, On some relations between the harmonic measure and the Lévy measure for a certain class of Markov processes, J. Math. Kyoto Univ. 2 (1962), 79-95.

[JK] D. S. Jerison and C. E. Kenig, Boundary value problems on Lipschitz domains, in: W. Littman (ed.), Studies in Partial Differential Equations, MAA Stud. Math. 23, Math. Assoc. Amer., Washington, DC, 1982, 1-68.

[J] A. Jonsson, Brownian motion on fractals and function spaces, Math. Z. 222 (1996), 495-504.

[JW] A. Jonsson and H. Wallin, Function Spaces on Subsets of $\mathbb{R}^{N}$, Math. Rep. 2, no. 1, Harwood, London, 1984.

[Kum] T. Kumagai, Some remarks for stable-like jump processes on fractals, in: Proc. Conf. held in Graz, 2001, Birkhäuser, 2002, 185-196.

[L] N. S. Landkof, Foundations of Modern Potential Theory, Springer, Berlin, 1969.

[P] K. Pietruska-Pałuba, On function spaces related to fractional diffusions on d-sets, Stochastics Stochastics Rep. 70 (2000), 153-164.

[PS] S. C. Port and C. J. Stone, Infinitely divisible processes and their potential theory, Ann. Inst. Fourier (Grenoble) 21 (1971), no. 2, 157-275, and no. 4, 179-265.

[R] M. Ryznar, Estimates of Green function for relativistic $\alpha$-stable process, Potential Anal. 17 (2002), 1-23.

[SW] R. Song and J. M. Wu, Boundary Harnack principle for symmetric stable processes, J. Funct. Anal. 168 (1999), 403-427.

[S] A. Stós, Symmetric stable processes on d-sets, Bull. Polish Acad. Sci. Math. 48 (2000), 237-245.

[Str] R. Strichartz, Analysis on fractals, Notices Amer. Math. Soc. 46 (1999), 11991208 .

Institute of Mathematics

Wrocław University of Technology

Wybrzeże Wyspiańskiego 27

50-370 Wrocław, Poland

E-mail: bogdan@im.pwr.wroc.pl

stos@im.pwr.wroc.pl

sztonyk@im.pwr.wroc.pl

Received September 30, 2002

Revised version March 21, 2003 